\title{
Solutions for $n$ th-order boundary value problems of impulsive singular nonlinear integro-differential equations in Banach spaces
}

\author{
Yanlai Chen ${ }^{1 *}$, Tingqiu Cao ${ }^{1}$ and Baoxia Qin ${ }^{2}$
}

\section{"Correspondence:}

yanlaichen@126.com

${ }^{1}$ School of Economics, Shandong

University, Jinan, Shandong 250100,

People's Republic of China

Full list of author information is

available at the end of the article

\begin{abstract}
Not considering the Green's function, the present study starts to construct a cone formed by a nonlinear term in Banach spaces, and through the cone creates a convex closed set. We obtain the existence of solutions for the boundary values problems of nth-order impulsive singular nonlinear integro-differential equations in Banach spaces by applying the Mönch fixed point theorem. An example is given to illustrate the main results.

MSC: 45J05; 34G20; 47H10

Keywords: impulsive singular integro-differential equation; Banach spaces; boundary value problem; Mönch fixed point theorem; measure of noncompactness
\end{abstract}

\section{Introduction and preliminaries}

By using the Schauder fixed point theorem, Guo [1] obtained the existence of solutions of initial value problems for $n$ th-order nonlinear impulsive integro-differential equations of mixed type on an infinite interval with infinite number of impulsive times in a Banach space. In [2], by using the fixed point theorem in a cone, Chen and Qin investigated the existence of multiple solutions for a class of boundary value problems of singular nonlinear integro-differential equations of mixed type in Banach spaces. For singular differential equations in Banach spaces please see [3-9]. Generally based on Green's function to construct a cone, but using the cone to study different nonlinear terms, we encountered difficulties, especially in infinite dimensional Banach spaces. In this paper, informed by the characteristics of the nonlinear term we construct a new cone, and through this cone create a convex closed set. On the new convex closed set, we apply the Mönch fixed point theorem to investigate the existence of solutions for the boundary value problems of $n$ thorder impulsive singular nonlinear integro-differential equations in Banach spaces. Finally, an example of scalar second-order impulsive integro-differential equations for an infinite system is offered. Because of difficulties of compactness arising from impulsiveness and the use of $n$ th-order integro-differential equations, a space $P C^{n-1}[J, E]$ is introduced. Let $E$ be a real Banach space and $J:=[0,1]$. Let $P C[J, E]:=\left\{u \mid u: J \rightarrow E u(t)\right.$ continuous at $t \neq t_{k}$, left continuous at $t=t_{k}$, and $u\left(t_{k}^{+}\right)$exists, $\left.k=1,2, \ldots, m\right\}$. Obviously $P C[J, E]$ is a Banach 
space with norm

$$
\|u\|:=\sup _{t \in J}\|u(t)\|
$$

Let $P C^{n-1}[J, E]:=\left\{u \in P C[J, E] \mid u^{(n-1)}(t)\right.$ exists and let it be continuous at $t \neq t_{k}$, let $u^{(n-1)}\left(t_{k}^{+}\right)$ and $u^{(n-1)}\left(t_{k}^{-}\right)$exist, $\left.k=1,2, \ldots, m\right\}$, where $u^{(n-1)}\left(t_{k}^{+}\right)$and $u^{(n-1)}\left(t_{k}^{-}\right)$represent the right and the left limits of $u^{(n-1)}(t)$ at $t=t_{k}$, respectively. For $u \in P C^{n-1}[J, E]$, we have

$$
\begin{gathered}
u^{(n-2)}\left(t_{k}-\epsilon\right)=u^{(n-2)}(t)+\int_{t}^{t_{k}-\epsilon} u^{(n-1)}(s) \mathrm{d} s, \\
\forall t_{k-1}<t<t_{k}-\epsilon<t_{k}(\epsilon>0), k=1,2, \ldots, m .
\end{gathered}
$$

So observing the existence of $u^{(n-1)}\left(t_{k}^{-}\right)$and taking limits as $\epsilon \rightarrow 0^{+}$in the above equality, we see that $u^{(n-2)}\left(t_{k}^{-}\right)$exists and

$$
u^{(n-2)}\left(t_{k}^{-}\right)=u^{(n-2)}(t)+\int_{t}^{t_{k}} u^{(n-1)}(s) \mathrm{d} s, \quad \forall t_{k-1}<t<t, k=1,2, \ldots, m .
$$

Similarly, we can show that $u^{(n-2)}\left(t_{k}^{+}\right)$exists. In the same way, we get the existence of $u^{(n-3)}\left(t_{k}^{-}\right), u^{(n-3)}\left(t_{k}^{+}\right), \ldots, u^{\prime}\left(t_{k}^{-}\right), u^{\prime}\left(t_{k}^{+}\right)$. Define $u^{(i)}\left(t_{k}\right)=u^{(i)}\left(t_{k}^{-}\right)(i=1,2, \ldots, n-1, k=$ $1,2, \ldots, m)$. Then $u^{(i)} \in P C[J, E](i=1,2, \ldots, n-1)$, and, as is natural, in the following, $u^{(i)}\left(t_{k}\right)$ is understood as $u^{(i)}\left(t_{k}^{-}\right)$. It is easy to see that $P C^{n-1}[J, E]$ is a Banach space with norm

$$
\|u\|_{P C^{n-1}}:=\max _{i=0,1, \ldots, n-1}\left\{\sup _{t \in J}\left\|u^{(i)}(t)\right\|\right\}
$$

Let $P$ be a cone in $E$ which defines a partial ordering in $E$ by $x \leq y$ if and only if $y-x \in P$. $P$ is said to be normal if there exists a positive constant $N$ such that $\theta \leq x \leq y$ implies $\|x\| \leq N\|y\|$, where the smallest $N$ is called the normal constant of $P$. For convenience, let $N=1$. Let $P_{1}=\left\{u \in P: u \geq u_{0}\|u\|\right\}$, in which $u_{0} \in P$ and $0<\left\|u_{0}\right\|<1$. For $r>0$, we write $P_{1 r}=\left\{u \in P_{1}:\|u\|<r\right\}$. We consider the following singular boundary value problem (SBVP for short) for an $n$ th-order impulsive nonlinear integro-differential equation in $E$ :

$$
\left\{\begin{array}{l}
-u^{(n)}(t)=f\left(t, u(t), u^{\prime}(t), \ldots, u^{(n-1)}(t),(T u)(t),(S u)(t)\right), \\
\quad 0<t<1, t \neq t_{k}(k=1,2, \ldots, m), \\
\left.\triangle u^{(i)}\right|_{t=t_{k}}=I_{i k}\left(u\left(t_{k}\right), u^{\prime}\left(t_{k}\right), \ldots, u^{(n-1)}\left(t_{k}\right)\right) \quad(i=0,1, \ldots, n-2 ; k=1,2, \ldots, m), \\
\left.\triangle u^{(n-1)}\right|_{t=t_{k}}=-I_{n-1 k}\left(u\left(t_{k}\right), u^{\prime}\left(t_{k}\right), \ldots, u^{(n-1)}\left(t_{k}\right)\right) \quad(k=1,2, \ldots, m), \\
u^{(i)}(0)=\theta \quad(i=0,1, \ldots, n-2), \quad u^{(n-1)}(1)=\theta
\end{array}\right.
$$

where $0<t_{1}<t_{2}<\cdots<t_{m}<1$,

$$
\begin{gathered}
f \in C[(0,1) \times \underbrace{P_{1} \backslash\{\theta\} \times P_{1} \backslash\{\theta\} \times \cdots \times P_{1} \backslash\{\theta\} \times P_{1} \times P_{1}}_{n+2}, P_{1}], \\
I_{i k} \in C \underbrace{P_{1} \times P_{1} \times \cdots \times P_{1}}_{n}, P_{1}](i=0,1, \ldots, n-1 ; k=1,2, \ldots, m), \text { and } \\
(T u)(t)=\int_{0}^{t} k(t, s) u(s) \mathrm{d} s, \quad(S u)(t)=\int_{0}^{1} h(t, s) u(s) \mathrm{d} s, \quad \forall t \in J,
\end{gathered}
$$


with $k \in C\left[D, R_{+}\right](D=\{(t, s) \in J \times J: t \geq s\}), h \in C\left[J \times J, R_{+}\right] .\left.\Delta u^{(i)}\right|_{t=t_{k}}$ denotes the jump of $u^{(i)}(t)$ at $t=t_{k}$, i.e.,

$$
\left.\Delta u^{(i)}\right|_{t=t_{k}}=u^{(i)}\left(t_{k}^{+}\right)-u^{(i)}\left(t_{k}^{-}\right)
$$

and $\theta$ denotes the zero element of $E$.

$f\left(t, v_{0}, v_{1}, \ldots, v_{n}, v_{n+1}\right)$ is singular at $v_{i}=\theta(i=0,1, \ldots, n-1), t=0$ and $/$ or $t=1$ if

$$
\lim _{v_{i} \rightarrow \theta}\left\|f\left(t, v_{0}, \ldots, v_{n+1}\right)\right\|=+\infty \quad(i=0,1, \ldots, n-1)
$$

$\forall t \in(0,1), v_{k} \in P_{1}(k=n, n+1), v_{j} \in P_{1} \backslash\{\theta\}(i, j=1, \ldots, n-1)$, and

$$
\lim _{t \rightarrow 0^{+}}\left\|f\left(t, v_{0}, \ldots, v_{n+1}\right)\right\|=+\infty \quad \text { and } / \text { or } \quad \lim _{t \rightarrow 1^{-}}\left\|f\left(t, v_{0}, \ldots, v_{n+1}\right)\right\|=+\infty
$$

$\forall v_{i} \in P_{1} \backslash\{\theta\}(i=0,1, \ldots, n-1), v_{j} \in P_{1}(j=n, n+1)$.

Remark Obviously, $P_{1} \subset P$, and $P_{1}$ is a normal cone of $E$ if $P$ is a normal cone of $E$. $P_{1}$ and $P$ has the same normal constant $N$.

In the following, we assume that $P$ is a normal cone. Let $J^{\prime}=J \backslash\left\{t_{1}, t_{2}, \ldots, t_{m}\right\}$. A map $u \in P C^{n-1}[J, E] \cap C^{n}\left[J^{\prime}, E\right]$ is called a solution of SBVP (1) if it satisfies (1).

\section{Several lemmas}

To continue, let us formulate some lemmas.

Lemma 2.1 If $H \subset P C^{n-1}[J, E]$ is bounded and the elements of $H^{(n-1)}$ are equicontinuous on each $J_{k}(k=0,1, \ldots, m)$, then

$$
\alpha_{n-1}(H)=\max _{i=0,1, \ldots, n-1}\left\{\sup _{t \in J} \alpha\left(H^{(i)}(t)\right)\right\}
$$

in which $\alpha$ denotes the Kuratowski measure of noncompactness, $H^{(i)}(t)=\left\{x^{(i)}(t): x \in H\right\}$ $(i=0,1, \ldots, n-1)$.

Proof For $i=0,1, \ldots, n-1$, it is easy to prove that

$$
\sup _{t \in J} \alpha\left(H^{(i)}(t)\right) \leq \alpha\left(H^{(i)}(J)\right) \leq \alpha\left(H^{(i)}\right)
$$

Since $\left\|u^{(i)}\right\| \leq\|u\|_{P C^{n-1}}(i=0,1, \ldots, n-1)$, we know $\alpha\left(H^{(i)}\right) \leq \alpha_{n-1}(H)(i=0,1, \ldots, n-1)$. Hence

$$
\max _{i=0,1, \ldots, n-1}\left\{\sup _{t \in J} \alpha\left(H^{(i)}(t)\right)\right\} \leq \alpha_{n-1}(H)
$$

Next, we check that

$$
\max _{i=0,1, \ldots, n-1}\left\{\sup _{t \in J} \alpha\left(H^{(i)}(t)\right)\right\} \geq \alpha_{n-1}(H) .
$$


In fact, for any $\epsilon>0$, there is a division $H^{(i)}=\bigcup_{l=1}^{p} H_{l}^{(i)}(i=0,1, \ldots, n-1)$ such that

$$
\operatorname{diam}\left(H_{l}^{(i)}\right)<\alpha\left(H^{(i)}\right)+\epsilon, \quad i=0,1, \ldots, n-1 .
$$

By hypothesis, the elements of $H^{(n-1)}$ are equicontinuous on each $J_{k}$ and there is a division:

$$
\begin{aligned}
0 & =t_{0}^{\prime}<t_{1}^{\prime}<\cdots<t_{j_{1}}^{\prime}=t_{1}<t_{j_{1}+1}^{\prime}<\cdots<t_{j_{2}}^{\prime} \\
& =t_{2}<\cdots<t_{j_{m}}^{\prime}=t_{m}<t_{j_{m}+1}^{\prime}<\cdots<t_{j_{m+1}}^{\prime}=1,
\end{aligned}
$$

such that

$$
\left\|u^{(i)}(t)-u^{(i)}\left(t_{1}^{\prime}\right)\right\|<\epsilon, \quad \forall u \in H, t \in\left[t_{0}^{\prime}, t_{1}^{\prime}\right](i=0,1, \ldots, n-1)
$$

and

$$
\left\|u^{(i)}(t)-u^{(i)}\left(t_{j}^{\prime}\right)\right\|<\epsilon, \quad \forall u \in H, t \in\left(t_{j-1}^{\prime}, t_{j}^{\prime}\right]\left(j=2, \ldots, j_{m+1}, i=0,1, \ldots, n-1\right)
$$

Let $J_{1}^{\prime}=\left[0, t_{1}^{\prime}\right], J_{j}^{\prime}=\left(t_{j-1}^{\prime}, t_{j}^{\prime}\right]\left(j=2, \ldots, j_{m+1}\right)$. By virtue of $(5)$ and $(6)$, we know that

$$
\left\|u^{(i)}(t)-u^{(i)}\left(t_{j}^{\prime}\right)\right\|<\epsilon, \quad \forall u \in H, t \in J_{j}^{\prime}\left(j=1,2, \ldots, j_{m+1}, i=0,1, \ldots, n-1\right) .
$$

Let $B:=\bigcup_{i=0}^{n-1} \bigcup_{j=1}^{j m+1} H^{(i)}\left(t_{j}^{\prime}\right)$. There is a division $B=\bigcup_{l=1}^{p} B_{l}$ such that

$$
\operatorname{diam} B_{l}<\alpha(B)+\epsilon \quad(l=1, \ldots, p) .
$$

Let $F$ be the finite set of all maps $\{0,1, \ldots, n-1\} \times\left\{1,2, \ldots, j_{m+1}\right\}$ into $\{1,2, \ldots, p\}(\mu:(i, j) \rightarrow$ $\mu(i, j))$. For $\mu \in F$, let $H_{\mu}:=\left\{u \in H: u^{(i)}\left(t_{j}^{\prime}\right) \in B_{\mu(i, j)},(i, j) \in\{0,1, \ldots, n-1\} \times\left\{1,2, \ldots, j_{m+1}\right\}\right\}$. It is clear that $H=\bigcup_{\mu \in F} H_{\mu}$. For any $u, v \in H_{\mu}, t \in J$, we have $t \in J_{j}^{\prime}$ for some $j \in\left\{1,2, \ldots, j_{m+1}\right\}$, and so

$$
\begin{aligned}
\left\|u^{(i)}(t)-v^{(i)}(t)\right\| & \leq\left\|u^{(i)}(t)-u^{(i)}\left(t_{j}^{\prime}\right)\right\|+\left\|u^{(i)}\left(t_{j}^{\prime}\right)-v^{(i)}\left(t_{j}^{\prime}\right)\right\|+\left\|v^{(i)}\left(t_{j}^{\prime}\right)-v^{(i)}(t)\right\| \\
& <\alpha(B)+3 \epsilon \quad(i=0,1, \ldots, n-1) .
\end{aligned}
$$

\section{Consequently,}

$$
\operatorname{diam} H_{\mu} \leq \alpha(B)+3 \epsilon, \quad \forall \mu \in F,
$$

which implies $\alpha_{n-1}(H) \leq \alpha(B)+3 \epsilon$. Since $\epsilon>0$ is arbitrary, we get

$$
\begin{aligned}
\alpha_{n-1}(H) & \leq \alpha(B)=\max \left\{\alpha\left(H^{(i)}\left(t_{j}^{\prime}\right)\right): j=1,2, \ldots, j_{m+1}, i=0,1, \ldots, n-1\right\} \\
& \leq \max _{i=0,1, \ldots, n-1}\left\{\sup _{t \in J} \alpha\left(H^{(i)}(t)\right)\right\} .
\end{aligned}
$$

Finally, the conclusion follows from (3) and (10). For details of the Kuratowski measure of noncompactness, please see [10]. 
Lemma 2.2 (see [11]) Let us take a countable set $D=\left\{u_{n}\right\} \subset L[J, E](n \in N)$. For all $u_{n} \in D$, there is $g \in L\left[J, R_{+}\right]$such that $\left\|u_{n}(t)\right\| \leq g(t)$, a.e, $t \in J$. Then $\alpha(D(t)) \in L\left[J, R_{+}\right]$, and

$$
\alpha\left(\left\{\int_{0}^{t} u_{n}(s) \mathrm{d} s: n \in N\right\}\right) \leq 2 \int_{0}^{t} \alpha(D(s)) \mathrm{d} s .
$$

Lemma 2.3 Suppose $H \subset P C[J, E]$ is bounded and equicontinuous on each $J_{k}$ ( $k=$ $0,1, \ldots, m)$. Then $\alpha(H(t)) \in P C\left[J, R_{+}\right]$, and

$$
\alpha\left(\left\{\int_{J} u(t) \mathrm{d} t: u \in H\right\}\right) \leq \int_{J} \alpha(H(t)) \mathrm{d} t .
$$

Proof By Theorem 1.2.2 of [10], the conclusion is obvious.

Lemma 2.4 Let $B_{1}, B_{2} \subset P C^{n-1}[J, E]$ be two countable sets. Suppose $u_{0} \in P C^{n-1}[J, E]$ and $\overline{B_{1}}=\overline{\mathrm{co}}\left(\left\{u_{0}\right\} \cup B_{2}\right)$. Then

$$
\overline{B_{1}^{(i)}(t)}=\overline{\mathrm{co}}\left(\left\{u_{0}^{(i)}(t)\right\} \cup B_{2}^{(i)}(t)\right), \quad t \in J(i=0,1, \ldots, n-1) .
$$

Proof The conclusion is obvious by Lemma 6 of [12].

Lemma 2.5 (see [13]) (The Mönch fixed point theorem) Let E be a Banach space. Assume that $D \subset E$ is close and convex. Assume also that $A: D \rightarrow D$ is continuous with the further property that for some $u_{0} \in D$, we have $C \subset D$ countable, $\bar{C}=\overline{\mathrm{co}}\left(\left\{u_{0}\right\} \cup A(C)\right)$ implies that $C$ is relatively compact. Then $A$ has a fixed-point in $D$.

\section{Main theorem and example}

For convenience, we list the following conditions:

$\left(\mathrm{H}_{1}\right)$ There exist $b \in C\left[J, R_{+}\right], a_{i} \in C\left[J, R_{+}\right](i=0,1, \ldots, n+1), g_{i} \in C[(0,+\infty),(0,+\infty)]$ $(i=0,1, \ldots, n-1)$ and $h_{i} \in C[[0,+\infty),[0,+\infty)](i=0,1, \ldots, n+1)$ such that

$$
\begin{aligned}
& \left\|f\left(t, v_{0}, v_{1}, \ldots, v_{n-1}, v_{n}, v_{n+1}\right)\right\| \\
& \leq b(t)+\sum_{i=0}^{n-1} a_{i}(t)\left(g_{i}\left(\left\|v_{i}\right\|\right)+h_{i}\left(\left\|v_{i}\right\|\right)\right)+a_{n}(t) h_{n}\left(\left\|v_{n}\right\|\right) \\
& \quad+a_{n+1}(t) h_{n+1}\left(\left\|v_{n+1}\right\|\right), \quad \forall t \in(0,1), v_{i} \in P_{1 r} \backslash\{\theta\}(i=0,1, \ldots, n-1), v_{n}, v_{n+1} \in P_{1 r},
\end{aligned}
$$

where $g_{i}$ is nonincreasing, $\frac{h_{i}}{g_{i}}(i=0,1, \ldots, n-1)$ and $h_{n}, h_{n+1}$ are nondecreasing. And there exist $d_{i k} \geq 0, c_{i k j} \geq 0(i, j=0,1, \ldots, n-1, k=1,2, \ldots, m)$ such that

$$
\left\|I_{i k}\left(v_{0}, v_{1}, \ldots, v_{n-1}\right)\right\| \leq d_{i k}+\sum_{j=0}^{n-1} c_{i k j}\left\|v_{j}\right\| \quad(i=0,1, \ldots, n-1, k=1,2, \ldots, m),
$$

$v_{j} \in P_{1 r}(j=0,1, \ldots, n-1)$.

$\left(\mathrm{H}_{2}\right)$ There exists a $\varphi \in P_{1}^{*}\left(P_{1}^{*}\right.$ denotes the dual cone of $\left.P_{1}\right)$ such that $\|\varphi\|=1$. And for any $r>0$, there exists a $h_{r}(t) \in L[(0,1),(0,+\infty)]$ such that

$$
\begin{aligned}
& \varphi\left(f\left(t, v_{0}, v_{1}, \ldots, v_{n-1}, v_{n}, v_{n+1}\right)\right) \\
& \quad \geq h_{r}(t), \quad \forall t \in(0,1), v_{i} \in P_{1 r} \backslash\{\theta\}(i=0,1, \ldots, n-1), v_{n}, v_{n+1} \in P_{1 r} .
\end{aligned}
$$


$\left(\mathrm{H}_{3}\right)$ There exists a $R_{0}>\int_{0}^{1} h_{R_{0}}(s) \mathrm{d} s$ such that

$$
\begin{aligned}
& \int_{0}^{1}\left(b(s)+\sum_{i=0}^{n-2} a_{i}(s) g_{i}\left(\varphi\left(u_{0}\right) \frac{s^{n-1-i}}{(n-1-i) !} \int_{0}^{1} \tau h_{R_{0}}(\tau) \mathrm{d} \tau\right)\left(1+\frac{h_{i}\left(R_{0}\right)}{g_{i}\left(R_{0}\right)}\right)+a_{n-1}(s)\right. \\
& \quad \times g_{n-1}\left(\varphi\left(u_{0}\right) \int_{s}^{1} h_{R_{0}}(\tau) \mathrm{d} \tau\right) \\
& \left.\quad \times\left(1+\frac{h_{n-1}\left(R_{0}\right)}{g_{n-1}\left(R_{0}\right)}\right)+a_{n}(s) h_{n}\left(k^{*} R_{0}\right)+a_{n+1}(s) h_{n+1}\left(h^{*} R_{0}\right)\right) \mathrm{d} s \\
& \quad+\sum_{k=1}^{m} \sum_{i=0}^{n-2} \frac{\left(1-t_{k}\right)^{i}}{i !}\left(d_{i k}+\sum_{j=0}^{n-1} c_{i k j} R_{0}\right)+\sum_{k=1}^{m}\left(d_{n-1 k}+\sum_{j=0}^{n-1} c_{n-1 k j} R_{0}\right) \leq R_{0},
\end{aligned}
$$

where $b, a_{i}(i=0,1, \ldots, n+1), g_{i}(i=0,1, \ldots, n-1), \varphi, h_{i}(i=0,1, \ldots, n+1), d_{i k}(i=0,1, \ldots, n-$ 1), $c_{i k j}(i, j=0,1, \ldots, n-1, k=0,1, \ldots, m)$ and $h_{R_{0}}$ are defined as in conditions $\left(\mathrm{H}_{1}\right)$ and $\left(\mathrm{H}_{2}\right)$, and $k^{*}:=\max _{(t, s) \in D}\{k(t, s)\}, h^{*}:=\max _{(t, s) \in J \times J}\{h(t, s)\}$.

$\left(\mathrm{H}_{4}\right)$ There exist $L_{i}(t) \in L\left[(0,1), R_{+}\right](i=0,1, \ldots, n+1), \forall b>a>0$ such that

$$
\alpha\left(f\left(t, B_{0}, B_{1}, \ldots, B_{n+1}\right)\right) \leq \sum_{i=0}^{n+1} L_{i}(t) \alpha\left(B_{i}\right), \quad \forall t \in(0,1),
$$

$B_{i} \subset \overline{P_{1 b}} \backslash P_{1 a}(i=0,1, \ldots, n-1), B_{n}, B_{n+1} \subset \overline{P_{1 b}}$. There exist $M_{i k j} \geq 0(i, j=0,1, \ldots, n-1$, $k=1,2, \ldots, m)$ such that

$$
\begin{aligned}
& \alpha\left(I_{i k}\left(B_{0}^{\prime}, B_{1}^{\prime}, \ldots, B_{n-1}^{\prime}\right)\right) \\
& \quad \leq \sum_{j=0}^{n-1} M_{i k j} \alpha\left(B_{j}^{\prime}\right), \quad B_{j}^{\prime} \subset \overline{P_{1 b}}(j=0,1, \ldots, n-1)(i=0,1, \ldots, n-1, k=1,2, \ldots, m) .
\end{aligned}
$$

Remark Obviously, condition $\left(\mathrm{H}_{4}\right)$ is satisfied automatically when $E$ is finite dimensional.

Lemma 3.1 Suppose conditions $\left(\mathrm{H}_{1}\right),\left(\mathrm{H}_{2}\right)$ and $\left(\mathrm{H}_{3}\right)$ are satisfied. Then $Q$ defined by

$$
\begin{aligned}
Q=: & \left\{u \in P C^{n-1}[J, P]: u^{(i)}(t) \geq u_{0}\left\|u^{(i)}(t)\right\|(i=0,1, \ldots, n-1),\left.\Delta u^{(i)}\right|_{t=t_{k}} \geq \theta\right. \\
& \left.(i=0,1, \ldots, n-2), \varphi\left(u^{(n-1)}(t)\right) \geq \varphi\left(u_{0}\right) \int_{t}^{1} h_{R_{0}}(s) \mathrm{d} s,\|u\|_{P C^{n-1}} \leq R_{0}, t \in J\right\}
\end{aligned}
$$

is a nonempty, convex and closed subset of $P C^{n-1}[J, E]$.

Proof Let

$$
\begin{aligned}
\tilde{u}(t)= & u_{0}\left(\frac{-1}{(n-1) !} \int_{0}^{t}(t-s)^{n-1} h_{R_{0}}(s) \mathrm{d} s+\frac{t^{n-1}}{(n-1) !} \int_{0}^{1} h_{R_{0}}(s) \mathrm{d} s\right. \\
& \left.+\sum_{0<t_{k}<t} \sum_{i=0}^{n-2} \frac{\left(t-t_{k}\right)^{i}}{i !}+m \frac{t^{n-1}}{(n-1) !}-\sum_{0<t_{k}<t} \frac{\left(t-t_{k}\right)^{n-1}}{(n-1) !}\right), \quad \forall t \in J .
\end{aligned}
$$


For $j=0,1, \ldots, n-1$,

$$
\begin{aligned}
\tilde{u}^{(j)}(t)= & u_{0}\left(\frac{-1}{(n-1-j) !} \int_{0}^{t}(t-s)^{n-1-j} h_{R_{0}}(s) \mathrm{d} s+\frac{t^{n-1-j}}{(n-1-j) !} \int_{0}^{1} h_{R_{0}}(s) \mathrm{d} s\right. \\
& \left.+\sum_{0<t_{k}<t} \sum_{i=j}^{n-2} \frac{\left(t-t_{k}\right)^{i-j}}{(i-j) !}+m \frac{t^{n-1-j}}{(n-1-j) !}-\sum_{0<t_{k}<t} \frac{\left(t-t_{k}\right)^{n-1-j}}{(n-1-j) !}\right), \quad \forall t \in J .
\end{aligned}
$$

It is clear that $\tilde{u}(t) \in P C^{n-1}[J, P]$. Since $0<\left\|u_{0}\right\|<1$, for $j=0,1, \ldots, n-1$, by (11), one can see that

$$
\begin{aligned}
\left\|\tilde{u}^{(j)}(t)\right\|= & \left\|u_{0}\right\| \mid \frac{-1}{(n-1-j) !} \int_{0}^{t}(t-s)^{n-1-j} h_{R_{0}}(s) \mathrm{d} s \\
& +\frac{t^{n-1-j}}{(n-1-j) !} \int_{0}^{1} h_{R_{0}}(s) \mathrm{d} s+\sum_{0<t_{k}<t} \sum_{i=j}^{n-2} \frac{\left(t-t_{k}\right)^{i-j}}{(i-j) !} \\
& +m \frac{t^{n-1-j}}{(n-1-j) !}-\sum_{0<t_{k}<t} \frac{\left(t-t_{k}\right)^{n-1-j}}{(n-1-j) !} \mid \\
\leq & \left(\frac{-1}{(n-1-j) !} \int_{0}^{t}(t-s)^{n-1-j} h_{R_{0}}(s) \mathrm{d} s+\frac{t^{n-1-j}}{(n-1-j) !} \int_{0}^{1} h_{R_{0}}(s) \mathrm{d} s\right. \\
& \left.+\sum_{0<t_{k}<t} \sum_{i=j}^{n-2} \frac{\left(t-t_{k}\right)^{i-j}}{(i-j) !}+m \frac{t^{n-1-j}}{(n-1-j) !}-\sum_{0<t_{k}<t} \frac{\left(t-t_{k}\right)^{n-1-j}}{(n-1-j) !}\right),
\end{aligned}
$$

which implies $\tilde{u}^{(i)}(t) \geq u_{0}\left\|\tilde{u}^{(i)}(t)\right\|(i=0,1, \ldots, n-1)$ for $t \in J$.

By conditions $\left(\mathrm{H}_{1}\right),\left(\mathrm{H}_{2}\right),\left(\mathrm{H}_{3}\right)$, and (11), we have

$$
\left.\triangle \tilde{u}^{(i)}\right|_{t=t_{k}} \geq \theta \quad(i=0,1, \ldots, n-2), \quad\|\tilde{u}\|_{P C^{n-1}} \leq R_{0}
$$

and $\varphi\left(\tilde{u}^{n-1}(t)\right) \geq \varphi\left(u_{0}\right) \int_{t}^{1} h_{R_{0}}(s) \mathrm{d} s$. Therefore, $\tilde{u} \in Q$ and $Q$ is a nonempty set.

Now, we check that $Q$ is a convex subset of $P C^{n-1}[J, E]$. In fact, for any $u, v \in Q, 0 \leq \lambda \leq 1$, we write $\tilde{v}=\lambda u+(1-\lambda) v$, which means $\tilde{v} \in P C^{n-1}[J, P]$. It is clear that

$$
\left.\Delta \tilde{\boldsymbol{v}}^{(i)}\right|_{t=t_{k}}=\left.\lambda \Delta u^{(i)}\right|_{t=t_{k}}+\left.(1-\lambda) \Delta v^{(i)}\right|_{t=t_{k}} \geq \lambda \theta+(1-\lambda) \theta=\theta \quad(i=0,1, \ldots, n-2) .
$$

By virtue of the characters of elements of $Q$ and the characters of $\varphi$, we have

$$
\begin{aligned}
\tilde{v}^{(i)}(t) & =\lambda u^{(i)}(t)+(1-\lambda) v^{(i)}(t) \geq \lambda u_{0}\left\|u^{(i)}(t)\right\|+(1-\lambda) u_{0}\left\|v^{(i)}(t)\right\| \\
& \geq u_{0}\left(\left\|\lambda u^{(i)}(t)+(1-\lambda) v^{(i)}(t)\right\|\right)=u_{0}\left\|\tilde{v}^{(i)}(t)\right\| \quad(i=0,1, \ldots, n-1) .
\end{aligned}
$$

In the same way,

$$
\begin{aligned}
\varphi\left(\tilde{v}^{(n-1)}\right) & =\varphi\left(\lambda u^{(n-1)}+(1-\lambda) v^{(n-1)}\right) \\
& \geq \lambda \varphi\left(u_{0}\right) \int_{t}^{1} h_{R_{0}}(s) \mathrm{d} s+(1-\lambda) \varphi\left(u_{0}\right) \int_{t}^{1} h_{R_{0}}(s) \mathrm{d} s=\varphi\left(u_{0}\right) \int_{t}^{1} h_{R_{0}}(s) \mathrm{d} s
\end{aligned}
$$


and

$$
\|\tilde{v}\|_{P C^{n-1}}=\|\lambda u+(1-\lambda) v\|_{P C^{n-1}} \leq \lambda R_{0}+(1-\lambda) R_{0}=R_{0} .
$$

Therefore, $\tilde{v} \in Q$. Thus, $Q$ is a convex subset of $P C^{n-1}[J, E]$. It is clear that $Q$ is a closed subset of $P C^{n-1}[J, E]$. So the conclusion holds.

Lemma 3.2 Assume that conditions $\left(\mathrm{H}_{1}\right),\left(\mathrm{H}_{2}\right)$ and $\left(\mathrm{H}_{3}\right)$ are satisfied. Then $A: Q \rightarrow Q$, where the operator $A$ is defined by

$$
\begin{aligned}
(A u)(t)= & \frac{-1}{(n-1) !} \int_{0}^{t}(t-s)^{n-1} f\left(s, u(s), u^{\prime}(s), \ldots, u^{(n-1)}(s),(T u)(s),(S u)(s)\right) \mathrm{d} s \\
& +\frac{t^{n-1}}{(n-1) !} \int_{0}^{1} f\left(s, u(s), u^{\prime}(s), \ldots, u^{(n-1)}(s),(T u)(s),(S u)(s)\right) \mathrm{d} s \\
& +\sum_{0<t_{k}<t} \sum_{i=0}^{n-2} \frac{\left(t-t_{k}\right)^{i}}{i !} I_{i k}\left(u\left(t_{k}\right), u^{\prime}\left(t_{k}\right), \ldots, u^{(n-1)}\left(t_{k}\right)\right) \\
& +\frac{t^{n-1}}{(n-1) !} \sum_{k=1}^{m} I_{n-1 k}\left(u\left(t_{k}\right), u^{\prime}\left(t_{k}\right), \ldots, u^{(n-1)}\left(t_{k}\right)\right) \\
& -\sum_{0<t_{k}<t} \frac{\left(t-t_{k}\right)^{n-1}}{(n-1) !} I_{n-1 k}\left(u\left(t_{k}\right), u^{\prime}\left(t_{k}\right), \ldots, u^{(n-1)}\left(t_{k}\right)\right), \quad \forall t \in J .
\end{aligned}
$$

Proof For any $u \in Q$, i.e.,

$$
u^{(i)}(t) \geq u_{0}\left\|u^{(i)}(t)\right\| \quad(i=0,1, \ldots, n-1)
$$

and

$$
\begin{aligned}
& \varphi\left(u^{(n-1)}(t)\right) \geq \varphi\left(u_{0}\right) \int_{t}^{1} h_{R_{0}}(s) \mathrm{d} s, \quad t \in J,\left.\Delta u^{(i)}\right|_{t=t_{k}} \geq \theta(i=0,1, \ldots, n-2), \\
& \|u\|_{P C^{n-1}} \leq R_{0} .
\end{aligned}
$$

For any $u \in Q$ and $t$ (fixed) $\in J$,

$$
\begin{aligned}
(T u)(t) & =\int_{0}^{t} k(t, s) u(s) \mathrm{d} s \geq \int_{0}^{t} k(t, s) u_{0}\|u(s)\| \mathrm{d} s \\
& \geq u_{0}\left\|\int_{0}^{t} k(t, s) u(s) \mathrm{d} s\right\|=u_{0}\|(T u)(t)\|
\end{aligned}
$$

and

$$
\begin{aligned}
(S u)(t) & =\int_{0}^{t} h(t, s) u(s) \mathrm{d} s \geq \int_{0}^{1} h(t, s) u_{0}\|u(s)\| \mathrm{d} s \\
& \geq u_{0}\left\|\int_{0}^{1} h(t, s) u(s) \mathrm{d} s\right\|=u_{0}\|(S u)(t)\| .
\end{aligned}
$$


Because of $\varphi \in P^{*},\|\varphi\|=1$ and $\varphi\left(u^{(n-1)}(t)\right) \geq \varphi\left(u_{0}\right) \int_{t}^{1} h_{R_{0}}(s) \mathrm{d} s, t \in(0,1)$, we know

$$
\left\|u^{(n-1)}(t)\right\|=\left\|u^{(n-1)}(t)\right\|\|\varphi\| \geq \varphi\left(u^{(n-1)}(t)\right) \geq \varphi\left(u_{0}\right) \int_{t}^{1} h_{R_{0}}(s) \mathrm{d} s .
$$

Analogously, for $i=0,1, \ldots, n-2$, it is easy to see

$$
\begin{aligned}
\varphi\left(u^{(j)}(t)\right)= & \varphi\left(\left.\sum_{0<t_{k}<t} \sum_{i=j}^{n-1} \frac{\left(t-t_{k}\right)^{i-j}}{(i-j) !} \Delta u^{(i)}\right|_{t=t_{k}}\right. \\
& \left.+\frac{1}{(n-2-j) !} \int_{0}^{t}(t-s)^{n-2-j} u^{(n-1)}(s) \mathrm{d} s\right) \\
\geq & \frac{1}{(n-2-j) !} \int_{0}^{t}(t-s)^{n-2-j} \varphi\left(u^{(n-1)}(s)\right) \mathrm{d} s \\
\geq & \frac{1}{(n-2-j) !} \int_{0}^{t}(t-s)^{n-2-j} \varphi\left(u_{0}\right) \int_{s}^{1} h_{R_{0}}(\tau) \mathrm{d} \tau \mathrm{d} s \\
\geq & \frac{t^{n-1-j}}{(n-1-j) !} \varphi\left(u_{0}\right) \int_{0}^{1}\left(1-(1-s)^{n-1-j}\right) h_{R_{0}}(s) \mathrm{d} s \\
\geq & \frac{t^{n-1-j}}{(n-1-j) !} \varphi\left(u_{0}\right) \int_{0}^{1} s h_{R_{0}}(s) \mathrm{d} s, \quad \forall t \in(0,1) .
\end{aligned}
$$

Hence,

$$
\left\|u^{(j)}(t)\right\| \geq \frac{t^{n-1-j}}{(n-1-j) !} \varphi\left(u_{0}\right) \int_{0}^{1} s h_{R_{0}}(s) \mathrm{d} s, \quad j=0,1, \ldots, n-2, \forall t \in(0,1) .
$$

Differentiating (15) $n-1$ times, we get

$$
\begin{aligned}
(A u)^{(n-1)}(t)= & \int_{t}^{1} f\left(s, u(s), u^{\prime}(s), \ldots, u^{(n-1)}(s),(T u)(s),(S u)(s)\right) \mathrm{d} s \\
& +\sum_{k=1}^{m} I_{n-1 k}\left(u\left(t_{k}\right), u^{\prime}\left(t_{k}\right), \ldots, u^{(n-1)}\left(t_{k}\right)\right) \\
& -\sum_{0<t_{k}<t} I_{n-1 k}\left(u\left(t_{k}\right), u^{\prime}\left(t_{k}\right), \ldots, u^{(n-1)}\left(t_{k}\right)\right), \quad \forall t \in J .
\end{aligned}
$$

Obviously, $(A u)^{(n-1)}\left(t_{i}^{+}\right)(i=1,2, \ldots, m)$ exist and

$$
\begin{aligned}
(A u)^{(n-1)}\left(t_{i}^{+}\right)= & \int_{t_{i}}^{1} f\left(s, u(s), u^{\prime}(s), \ldots, u^{(n-1)}(s),(T u)(s),(S u)(s)\right) \mathrm{d} s \\
& +\sum_{k=i+1}^{m} I_{n-1 k}\left(u\left(t_{k}\right), u^{\prime}\left(t_{k}\right), \ldots, u^{(n-1)}\left(t_{k}\right)\right), \quad \forall t \in J(i=1,2, \ldots, m),
\end{aligned}
$$

where $\sum_{k=i+1}^{m} I_{n-1 k}\left(u\left(t_{k}\right), u^{\prime}\left(t_{k}\right), \ldots, u^{(n-1)}\left(t_{k}\right)\right)$ is understood as $\theta$ for $i=m$. Similarly, $(A u)^{(n-1)}\left(t_{i}^{-}\right)(i=1,2, \ldots, m)$ exist. Hence,

$$
A u \in P C^{n-1}[J, P]
$$


Let

$$
G_{l}(t, s)=:\left\{\begin{array}{ll}
\frac{t^{n-1-l}-(t-s)^{n-1-l}}{(n-1-l) !}, & 0 \leq s \leq t \leq 1 ; \\
\frac{t^{n-1-l}}{(n-1-l) !}, & 0 \leq t<s \leq 1
\end{array} \quad(l=0,1, \ldots, n-1) .\right.
$$

Since

$$
f \in C[(0,1) \times \underbrace{P_{1} \backslash\{\theta\} \times P_{1} \backslash\{\theta\} \times \cdots \times P_{1} \backslash\{\theta\} \times P_{1} \times P_{1}}_{n+2}, P_{1}]
$$

and $I_{i k} \in C[\underbrace{P_{1} \times P_{1} \times \cdots \times P_{1}}, P_{1}](i=0,1, \ldots, n-1 ; k=1,2, \ldots, m)$, it follows from (15), (16), (17) and (18) that

$$
\begin{aligned}
& (A u)^{(l)}(t)=\int_{0}^{1} G_{l}(t, s) f\left(u(s), u^{\prime}(s), \ldots, u^{(n-1)}(s),(T u)(s),(S u)(s)\right) \mathrm{d} s \\
& +\sum_{0<t_{k}<t} \sum_{i=l}^{n-2} \frac{\left(t-t_{k}\right)^{i-l}}{(i-l) !} I_{i k}\left(u\left(t_{k}\right), u^{\prime}\left(t_{k}\right), \ldots, u^{(n-1)}\left(t_{k}\right)\right) \\
& +\frac{t^{n-1-l}}{(n-1-l) !} \sum_{k=1}^{m} I_{n-1 k}\left(u\left(t_{k}\right), u^{\prime}\left(t_{k}\right), \ldots, u^{(n-1)}\left(t_{k}\right)\right) \\
& -\sum_{0<t_{k}<t} \frac{\left(t-t_{k}\right)^{n-1-l}}{(n-1-l) !} I_{n-1 k}\left(u\left(t_{k}\right), u^{\prime}\left(t_{k}\right), \ldots, u^{(n-1)}\left(t_{k}\right)\right) \\
& \geq \int_{0}^{1} G_{l}(t, s) u_{0}\left\|f\left(u(s), u^{\prime}(s), \ldots, u^{(n-1)}(s),(T u)(s),(S u)(s)\right)\right\| \mathrm{d} s \\
& +\sum_{0<t_{k}<t} \sum_{i=l}^{n-2} \frac{\left(t-t_{k}\right)^{i-l}}{(i-l) !} u_{0}\left\|I_{i k}\left(u\left(t_{k}\right), u^{\prime}\left(t_{k}\right), \ldots, u^{(n-1)}\left(t_{k}\right)\right)\right\| \\
& +\left(\frac{t^{n-1-l}}{(n-1-l) !} \sum_{k=1}^{m} 1_{k}-\sum_{0<t_{k}<t} \frac{\left(t-t_{k}\right)^{n-1-l}}{(n-1-l) !}\right) \\
& \times u_{0}\left\|I_{n-1 k}\left(u\left(t_{k}\right), u^{\prime}\left(t_{k}\right), \ldots, u^{(n-1)}\left(t_{k}\right)\right)\right\| \\
& \geq u_{0} \| \int_{0}^{1} G_{l}(t, s) f\left(u(s), u^{\prime}(s), \ldots, u^{(n-1)}(s),(T u)(s),(S u)(s)\right) \mathrm{d} s \\
& +\sum_{0<t_{k}<t} \sum_{i=l}^{n-2} \frac{\left(t-t_{k}\right)^{i-l}}{(i-l) !} I_{i k}\left(u\left(t_{k}\right), u^{\prime}\left(t_{k}\right), \ldots, u^{(n-1)}\left(t_{k}\right)\right) \\
& +\frac{t^{n-1-l}}{(n-1-l) !} \sum_{k=1}^{m} I_{n-1 k}\left(u\left(t_{k}\right), u^{\prime}\left(t_{k}\right), \ldots, u^{(n-1)}\left(t_{k}\right)\right) \\
& -\sum_{0<t_{k}<t} \frac{\left(t-t_{k}\right)^{n-1-l}}{(n-1-l) !} I_{n-1 k}\left(u\left(t_{k}\right), u^{\prime}\left(t_{k}\right), \ldots, u^{(n-1)}\left(t_{k}\right)\right) \| \\
& =u_{0}\left\|(A u)^{(l)}(t)\right\|, \quad l=0,1, \ldots, n-1, \forall t \in J .
\end{aligned}
$$

It is clear that

$$
\left.\Delta(A u)^{(l)}\right|_{t=t_{k}} \geq \theta, \quad l=0,1, \ldots, n-2 .
$$


Chen et al. Boundary Value Problems 2014, 2014:128

Page 11 of 25

Since $\varphi\left(u_{0}\right) \leq\|\varphi\|\left\|u_{0}\right\| \leq 1$, by (22), (26) and condition $\left(\mathrm{H}_{2}\right)$, we have

$$
\begin{aligned}
\varphi\left((A u)^{(n-1)}(t)\right)= & \varphi\left(\int_{t}^{1} f\left(s, u(s), u^{\prime}(s), \ldots, u^{(n-1)}(s),(T u)(s),(S u)(s)\right) \mathrm{d} s\right. \\
& +\sum_{k=1}^{m} I_{n-1 k}\left(u\left(t_{k}\right), u^{\prime}\left(t_{k}\right), \ldots, u^{(n-1)}\left(t_{k}\right)\right) \\
& \left.-\sum_{0<t_{k}<t} I_{n-1 k}\left(u\left(t_{k}\right), u^{\prime}\left(t_{k}\right), \ldots, u^{(n-1)}\left(t_{k}\right)\right)\right) \\
\geq & \int_{t}^{1} h_{R_{0}}(s) \mathrm{d} s \geq \varphi\left(u_{0}\right) \int_{t}^{1} h_{R_{0}}(s) \mathrm{d} s, \quad \forall t \in J .
\end{aligned}
$$

Now, we show that

$$
\|A u\|_{P C^{n-1}} \leq R_{0}, \quad \forall u \in Q
$$

By (15), (19), (21), conditions $\left(\mathrm{H}_{1}\right)$ and $\left(\mathrm{H}_{3}\right)$ imply

$$
\begin{aligned}
& \left\|(A u)^{(l)}(t)\right\|=\| \int_{0}^{1} G_{l}(t, s) f\left(u(s), u^{\prime}(s), \ldots, u^{(n-1)}(s),(T u)(s),(S u)(s)\right) \mathrm{d} s \\
& +\sum_{0<t_{k}<t} \sum_{i=l}^{n-2} \frac{\left(t-t_{k}\right)^{i-l}}{(i-l) !} I_{i k}\left(u\left(t_{k}\right), u^{\prime}\left(t_{k}\right), \ldots, u^{(n-1)}\left(t_{k}\right)\right) \\
& +\frac{t^{n-1-l}}{(n-1-l) !} \sum_{k=1}^{m} I_{n-1 k}\left(u\left(t_{k}\right), u^{\prime}\left(t_{k}\right), \ldots, u^{(n-1)}\left(t_{k}\right)\right) \\
& -\sum_{0<t_{k}<t} \frac{\left(t-t_{k}\right)^{n-1-l}}{(n-1-l) !} I_{n-1 k}\left(u\left(t_{k}\right), u^{\prime}\left(t_{k}\right), \ldots, u^{(n-1)}\left(t_{k}\right)\right) \| \\
& \leq \int_{0}^{1}\left\|G_{l}(t, s) f\left(u(s), u^{\prime}(s), \ldots, u^{(n-1)}(s),(T u)(s),(S u)(s)\right)\right\| \mathrm{d} s \\
& +\| \sum_{0<t_{k}<t} \sum_{i=l}^{n-2} \frac{\left(t-t_{k}\right)^{i-l}}{(i-l) !} I_{i k}\left(u\left(t_{k}\right), u^{\prime}\left(t_{k}\right), \ldots, u^{(n-1)}\left(t_{k}\right)\right) \\
& +\frac{t^{n-1-l}}{(n-1-l) !} \sum_{k=1}^{m} I_{n-1 k}\left(u\left(t_{k}\right), u^{\prime}\left(t_{k}\right), \ldots, u^{(n-1)}\left(t_{k}\right)\right) \\
& -\sum_{0<t_{k}<t} \frac{\left(t-t_{k}\right)^{n-1-l}}{(n-1-l) !} I_{n-1 k}\left(u\left(t_{k}\right), u^{\prime}\left(t_{k}\right), \ldots, u^{(n-1)}\left(t_{k}\right)\right) \| \\
& \leq \int_{0}^{1}\left(b(s)+\sum_{i=0}^{n-2} a_{i}(s) g_{i}\left(\varphi\left(u_{0}\right) \frac{s^{n-1-i}}{(n-1-i) !} \int_{0}^{1} \tau h_{R_{0}}(\tau) \mathrm{d} \tau\right)\left(1+\frac{h_{i}\left(R_{0}\right)}{g_{i}\left(R_{0}\right)}\right)\right. \\
& +a_{n-1}(s) g_{n-1}\left(\varphi\left(u_{0}\right) \int_{s}^{1} h_{R_{0}}(\tau) \mathrm{d} \tau\right)\left(1+\frac{h_{n-1}\left(R_{0}\right)}{g_{n-1}\left(R_{0}\right)}\right) \\
& \left.+a_{n}(s) h_{n}\left(k^{*} R_{0}\right)+a_{n+1}(s) h_{n+1}\left(h^{*} R_{0}\right)\right) \mathrm{d} s
\end{aligned}
$$




$$
\begin{aligned}
& \quad+\sum_{k=1}^{m} \sum_{i=0}^{n-2} \frac{\left(1-t_{k}\right)^{i}}{i !}\left(d_{i k}+\sum_{j=0}^{n-1} c_{i k j} R_{0}\right)+\sum_{k=1}^{m}\left(d_{n-1 k}+\sum_{j=0}^{n-1} c_{n-1 k j} R_{0}\right) \\
& \leq R_{0}, \quad l=0,1, \ldots, n-1, \forall t \in J,
\end{aligned}
$$

which implies that (29) is true. By (24), (26) to (29), the conclusion holds.

Lemma 3.3 Suppose conditions $\left(\mathrm{H}_{1}\right)$ to $\left(\mathrm{H}_{4}\right)$ are satisfied. Let

$$
\begin{aligned}
D_{l}(t) & =\left\{\int_{0}^{1} G_{l}(t, s) f\left(s, u(s), \ldots, u^{(n-1)}(s),(T u)(s),(S u)(s)\right) \mathrm{d} s: u \in B\right\} \\
(l & =0,1, \ldots, n-2)
\end{aligned}
$$

and

$$
D_{n-1}(t)=\left\{\int_{t}^{1} f\left(s, u(s), \ldots, u^{(n-1)}(s),(T u)(s),(S u)(s)\right) \mathrm{d} s: u \in B\right\}
$$

in which $t \in J, B($ countable $) \subset Q$. Then

$$
\begin{aligned}
& \alpha\left(D_{l}(t)\right) \leq \int_{0}^{1} 2 s\left(\sum_{i=0}^{n-1} L_{i}(s) \alpha\left(B^{(i)}(s)\right)+L_{n}(s) k^{*} \alpha(B(s))+L_{n+1}(s) h^{*} \alpha(B(s))\right) \mathrm{d} s \\
& (l=0,1, \ldots, n-2)
\end{aligned}
$$

and

$$
\alpha\left(D_{n-1}(t)\right) \leq \int_{0}^{1} 2\left(\sum_{i=0}^{n-1} L_{i}(s) \alpha\left(B^{(i)}(s)\right)+L_{n}(s) k^{*} \alpha(B(s))+L_{n+1}(s) h^{*} \alpha(B(s))\right) \mathrm{d} s,
$$

in which $B^{(i)}(s)=\left\{u^{(i)}(s): u \in B\right\}(i=0,1, \ldots, n-1)$.

Proof In order to avoid the singularity, given $\frac{1}{2}>\delta>0$, let

$$
\begin{gathered}
D_{l \delta}(t)=:\left\{\int_{\delta}^{1-\delta} G_{l}(t, s) f\left(s, u(s), \ldots, u^{(n-1)}(s),(T u)(s),(S u)(s)\right) \mathrm{d} s: u \in B\right\} \\
(l=0,1, \ldots, n-2), 0<\delta<\frac{1}{2}, t \in J .
\end{gathered}
$$

By conditions $\left(\mathrm{H}_{1}\right),\left(\mathrm{H}_{2}\right)$ and $\left(\mathrm{H}_{3}\right)$, for any $t \in J, u \in B$, we have

$$
\begin{aligned}
& \| \int_{0}^{1} G_{l}(t, s) f\left(s, u(s), \ldots, u^{(n-1)}(s),(T u)(s),(S u)(s)\right) \mathrm{d} s \\
& \quad-\int_{\delta}^{1-\delta} G_{l}(t, s) f\left(s, u(s), \ldots, u^{(n-1)}(s),(T u)(s),(S u)(s)\right) \mathrm{d} s \| \\
& \quad \leq \int_{0}^{\delta}\left(b(s)+\sum_{i=0}^{n-2} a_{i}(s) g_{i}\left(\varphi\left(u_{0}\right) \frac{s^{n-1-i}}{(n-1-i) !} \int_{0}^{1} \tau h_{R_{0}}(\tau) \mathrm{d} \tau\right)\left(1+\frac{h_{i}\left(R_{0}\right)}{g_{i}\left(R_{0}\right)}\right)\right. \\
& \quad+a_{n-1}(s) g_{n-1}\left(\varphi\left(u_{0}\right) \int_{s}^{1} h_{R_{0}}(\tau) \mathrm{d} \tau\right)
\end{aligned}
$$




$$
\begin{aligned}
& \left.\times\left(1+\frac{h_{n-1}\left(R_{0}\right)}{g_{n-1}\left(R_{0}\right)}\right)+a_{n}(s) h_{n}\left(k^{*} R_{0}\right)+a_{n+1}(s) h_{n+1}\left(h^{*} R_{0}\right)\right) \mathrm{d} s \\
& +\int_{1-\delta}^{1}\left(b(s)+\sum_{i=0}^{n-2} a_{i}(s) g_{i}\left(\varphi\left(u_{0}\right) \frac{s^{n-1-i}}{(n-1-i) !} \int_{0}^{1} \tau h_{R_{0}}(\tau) \mathrm{d} \tau\right)\left(1+\frac{h_{i}\left(R_{0}\right)}{g_{i}\left(R_{0}\right)}\right)\right. \\
& +a_{n-1}(s) g_{n-1}\left(\varphi\left(u_{0}\right) \int_{s}^{1} h_{R_{0}}(\tau) \mathrm{d} \tau\right)\left(1+\frac{h_{n-1}\left(R_{0}\right)}{g_{n-1}\left(R_{0}\right)}\right) \\
& \left.+a_{n}(s) h_{n}\left(k^{*} R_{0}\right)+a_{n+1}(s) h_{n+1}\left(h^{*} R_{0}\right)\right) \mathrm{d} s .
\end{aligned}
$$

By virtue of absolute continuity of the Lebesgue integrable function, we have

$$
d_{H\left(D_{l \delta}(t), D_{l}(t)\right)} \rightarrow 0, \quad \text { as } \delta \rightarrow 0, \forall t \in J,
$$

in which, $d_{H\left(D_{l \delta}(t), D_{l}(t)\right)}$ denotes the Hausdorff distance between $D_{l \delta}(t)$ and $D_{l}(t)$. Therefore,

$$
\alpha\left(D_{l}(t)\right)=\lim _{\delta \rightarrow 0} \alpha\left(D_{l \delta}(t)\right), \quad \forall t \in J .
$$

Now, we show

$$
\begin{aligned}
& \alpha\left(D_{l \delta}(t)\right) \leq \int_{0}^{1} 2 s\left(\sum_{i=0}^{n-1} L_{i}(s) \alpha\left(B^{(i)}(s)\right)+L_{n}(s) k^{*} \alpha(B(s))+L_{n+1}(s) h^{*} \alpha(B(s))\right) \mathrm{d} s \\
& (l=0,1, \ldots, n-1) .
\end{aligned}
$$

In fact, by Lemma 2.2, we have

$$
\begin{aligned}
\alpha\left(D_{l \delta}(t)\right)= & \alpha\left(\left\{\int_{\delta}^{1-\delta} G_{l}(t, s) f\left(s, u(s), \ldots, u^{(n-1)}(s),(T u)(s),(S u)(s)\right) \mathrm{d} s: u \in B\right\}\right) \\
\leq & \int_{\delta}^{1-\delta} 2 G_{l}(t, s) \alpha\left(f\left(s, B(s), \ldots, B^{(n-1)}(s),(T B)(s),(S B)(s)\right)\right) \mathrm{d} s \\
& (l=0,1, \ldots, n-2),
\end{aligned}
$$

where $(T B)=\{(T u)(t): u \in B\},(S B)=\{(S u)(t): u \in B\}$.

On the other hand, for $u \in B \subset Q$, it follows from (19) and (21) that

$$
\left\|u^{(j)}(s)\right\| \geq \frac{\delta^{n-1-j}}{(n-1-j) !} \varphi\left(u_{0}\right) \int_{0}^{1} s h_{R_{0}}(s) \mathrm{d} s, \quad j=0,1, \ldots, n-2, \forall s \in(\delta, 1-\delta)
$$

and

$$
\left\|u^{(n-1)}(s)\right\| \geq \varphi\left(u_{0}\right) \int_{1-\delta}^{1} h_{R_{0}}(s) \mathrm{d} s, \quad s \in(\delta, 1-\delta) .
$$

Taking $a=\min \left\{\min _{j=0,1, \ldots, n-2}\left\{\frac{\delta^{n-1-j}}{(n-1-j) !} \varphi\left(u_{0}\right) \int_{0}^{1} h_{R_{0}}(s) \mathrm{d} s\right\}, \frac{\delta^{n-1-j}}{(n-1-j) !} \varphi\left(u_{0}\right) \int_{0}^{1} h_{R_{0}}(s) \mathrm{d} s\right\}, b=\max \left\{k^{*}\right.$, $\left.h^{*}, 1\right\} R_{0}$, by (16), (17) and (18), one can see that

$$
B^{(i)}(s) \subset \overline{P_{1 b}} \backslash P_{1 a} \quad(i=0,1, \ldots, n-1), \quad(T B)(s),(S B)(s) \subset \overline{P_{1 b}} .
$$


Therefore, by condition $\left(\mathrm{H}_{4}\right)$ and (36), for $l=0,1, \ldots, n-2$, it is easy to get

$$
\begin{aligned}
& \alpha\left(D_{l \delta}(t)\right) \leq \int_{0}^{1} 2 G_{l}(t, s)\left(\sum_{i=0}^{n-1} L_{i}(s) \alpha\left(B^{i}(s)\right)+L_{n}(s) \alpha((T B)(s))+L_{n+1}(s) \alpha((S B)(s))\right) \mathrm{d} s, \\
& \quad t \in J .
\end{aligned}
$$

Since $B$ is a bounded set of $P C^{n-1}[J, E]$ and $B^{\prime}(t)$ is a bounded set, $B(t)$ is equicontinuous on each $J_{k}(k=1,2, \ldots, m)$. By Lemma 2.3 , it is easy to get

$$
\alpha((T B)(s)) \leq k^{*} \int_{0}^{s} \alpha(B(\tau)) \mathrm{d} \tau, \quad \alpha((S B)(s)) \leq h^{*} \int_{0}^{1} \alpha(B(\tau)) \mathrm{d} \tau .
$$

Substituting (41) into (40), we get (31).

Similarly, we obtain (32) and our conclusion holds.

Lemma 3.4 Let conditions $\left(\mathrm{H}_{1}\right)$ to $\left(\mathrm{H}_{3}\right)$ be satisfied. $u \in P C^{n-1}[J, E] \cap C^{n}\left[J^{\prime}, E\right]$ is a solution of $S B V P(1)$, if and only if $u \in Q$ is a fixed point of the operator A defined by (15).

Proof First of all, by mathematical induction, for $u \in P C^{n-1}[J, E] \cap C^{n}\left[J^{\prime}, E\right]$, Taylor's formula with the integral remainder term holds,

$$
\begin{aligned}
u(t)= & \sum_{i=0}^{n-1} \frac{t^{i}}{i !} u^{(i)}(0)+\sum_{0<t_{k}<t} \sum_{i=0}^{n-1} \frac{\left(t-t_{k}\right)^{i}}{i !}\left[u^{(i)}\left(t_{k}^{+}\right)-u^{(i)}\left(t_{k}\right)\right] \\
& +\frac{1}{(n-1) !} \int_{0}^{t}(t-s)^{n-1} u^{(n)}(s) \mathrm{d} s .
\end{aligned}
$$

In fact, as $n=1$, for $u \in P C[J, E] \cap C^{1}\left[J^{\prime}, E\right]$, let $t_{k}<t \leq t_{k+1}$, it is easy to see that

$$
\begin{aligned}
& u\left(t_{1}\right)-u(0)=\int_{0}^{t_{1}} u^{\prime}(s) \mathrm{d} s, \quad u\left(t_{2}\right)-u\left(t_{1}^{+}\right)=\int_{t_{1}}^{t_{2}} u^{\prime}(s) \mathrm{d} s, \\
& \ldots, \\
& u\left(t_{k}\right)-u\left(t_{k-1}^{+}\right)=\int_{t_{k-1}}^{t_{k}} u^{\prime}(s) \mathrm{d} s, \quad u(t)-u\left(t_{k}^{+}\right)=\int_{t_{k}}^{t} u^{\prime}(s) \mathrm{d} s .
\end{aligned}
$$

Adding these together, we get

$$
u(t)-u(0)-\sum_{i=1}^{k}\left[u\left(t_{i}^{+}\right)-u\left(t_{i}\right)\right]=\int_{0}^{t} u^{\prime}(s) \mathrm{d} s
$$

that is,

$$
u(t)=u(0)+\sum_{0<t_{k}<t}\left[u\left(t_{k}^{+}\right)-u\left(t_{k}\right)\right]+\int_{0}^{t} u^{\prime}(s) \mathrm{d} s, \quad \forall t \in J .
$$

This proves that (42) is true for $n=1$. 
Suppose (42) is true for $n-1$, i.e., for $u \in P C^{n-2}[J, E] \cap C^{n-1}\left[U^{\prime}, E\right]$, the next formula holds:

$$
\begin{aligned}
u(t)= & \sum_{i=0}^{n-2} \frac{t^{i}}{i !} u^{(i)}(0)+\sum_{0<t_{k}<t} \sum_{i=0}^{n-2} \frac{\left(t-t_{k}\right)^{i}}{i !}\left[u^{(i)}\left(t_{k}^{+}\right)-u^{(i)}\left(t_{k}\right)\right] \\
& +\frac{1}{(n-2) !} \int_{0}^{t}(t-s)^{n-2} u^{(n-1)}(s) \mathrm{d} s .
\end{aligned}
$$

Now we check that (42) is also true for $n$. In fact, suppose $u \in P C^{n-1}[J, E] \cap C^{n}\left[J^{\prime}, E\right]$. Then $u^{(n-1)} \in P C[J, E] \cap C^{1}\left[J^{\prime}, E\right]$, by (43), one can see

$$
u^{(n-1)}(t)=u^{(n-1)}(0)+\sum_{0<t_{k}<t}\left[u^{(n-1)}\left(t_{k}^{+}\right)-u^{(n-1)}\left(t_{k}\right)\right]+\int_{0}^{t} u^{(n)}(s) \mathrm{d} s, \quad \forall t \in J .
$$

Substituting the above equation into (44), we get

$$
\begin{aligned}
u(t)= & \sum_{i=0}^{n-2} \frac{t^{i}}{i !} u^{(i)}(0)+\sum_{0<t_{k}<t} \sum_{i=0}^{n-2} \frac{\left(t-t_{k}\right)^{i}}{i !}\left[u^{(i)}\left(t_{k}^{+}\right)-u^{(i)}\left(t_{k}\right)\right] \\
& +\frac{1}{(n-2) !} \int_{0}^{t}(t-s)^{n-2} \\
& \times\left\{u^{(n-1)}(0)+\sum_{0<t_{k}<s}\left[u^{(n-1)}\left(t_{k}^{+}\right)-u^{(n-1)}\left(t_{k}\right)\right]+\int_{0}^{s} u^{(n)}(\tau) \mathrm{d} \tau\right\} \mathrm{d} s \\
= & \sum_{i=0}^{n-2} \frac{t^{i}}{i !} u^{(i)}(0)+\sum_{0<t_{k}<t} \sum_{i=0}^{n-2} \frac{\left(t-t_{k}\right)^{i}}{i !}\left[u^{(i)}\left(t_{k}^{+}\right)-u^{(i)}\left(t_{k}\right)\right]+\left.\frac{(t-s)^{n-1}}{(n-1) !}\right|_{t} ^{0} u^{(n-1)}(0) \\
& -\sum_{0<t_{k}<t} \int_{t_{k}}^{t} \frac{(t-s)^{n-2}}{(n-2) !}\left[u^{(n-1)}\left(t_{k}^{+}\right)-u^{(n-1)}\left(t_{k}\right)\right] \mathrm{d} s+\frac{1}{(n-1) !} \int_{0}^{t}(t-s)^{n-1} u^{(n)}(s) \mathrm{d} s \\
= & \sum_{i=0}^{n-1} \frac{t^{i}}{i !} u^{(i)}(0)+\sum_{0<t_{k}<t} \sum_{i=0}^{n-1} \frac{\left(t-t_{k}\right)^{i}}{i !}\left[u^{(i)}\left(t_{k}^{+}\right)-u^{(i)}\left(t_{k}\right)\right] \\
& +\frac{1}{(n-1) !} \int_{0}^{t}(t-s)^{n-1} u^{(n)}(s) \mathrm{d} s, \quad \forall t \in J .
\end{aligned}
$$

So, (42) is also true for $n$. By mathematical induction, (42) holds.

Suppose $u \in P C^{n-1}[J, E] \cap C^{n}\left[J^{\prime}, E\right]$ is a solution of SBVP (1). By (42), we can see that

$$
\begin{aligned}
u(t)= & \sum_{i=0}^{n-1} \frac{t^{i}}{i !} u^{(i)}(0)+\sum_{0<t_{k}<t} \sum_{i=0}^{n-1} \frac{\left(t-t_{k}\right)^{i}}{i !}\left[u^{(i)}\left(t_{k}^{+}\right)-u^{(i)}\left(t_{k}\right)\right] \\
& +\frac{1}{(n-1) !} \int_{0}^{t}(t-s)^{n-1} u^{(n)}(s) \mathrm{d} s .
\end{aligned}
$$

Substituting

$$
u^{(n-1)}(0)=u^{(n-1)}(a)-\int_{0}^{a} u^{(n)}(s) \mathrm{d} s-\sum_{k=1}^{m}\left[u^{(n-1)}\left(t_{k}^{+}\right)-u^{(n-1)}\left(t_{k}\right)\right]
$$


into (47), by (15), we get $u(t)=(A u)(t)$. So $u$ is a fixed point of the operator $A$ defined by (15) in $Q$.

Conversely, if $u \in Q$ is a fixed point of the operator $A$, i.e., $u$ is a solution of the following impulsive integro-differential equation:

$u(t)=(A u)(t)$.

Then, by (15), similar to (26), from the derivative of both sides of the above equation one can draw the following conclusions:

$$
\begin{aligned}
u^{(l)}(t)= & \int_{0}^{1} G_{l}(t, s) f\left(u(s), u^{\prime}(s), \ldots, u^{(n-1)}(s),(T u)(s),(S u)(s)\right) \mathrm{d} s \\
& +\sum_{0<t_{k}<t} \sum_{i=l}^{n-2} \frac{\left(t-t_{k}\right)^{i-l}}{(i-l) !} I_{i k}\left(u\left(t_{k}\right), u^{\prime}\left(t_{k}\right), \ldots, u^{(n-1)}\left(t_{k}\right)\right) \\
& +\frac{t^{n-1-l}}{(n-1-l) !} \sum_{k=1}^{m} I_{n-1 k}\left(u\left(t_{k}\right), u^{\prime}\left(t_{k}\right), \ldots, u^{(n-1)}\left(t_{k}\right)\right) \\
& -\sum_{0<t_{k}<t} \frac{\left(t-t_{k}\right)^{n-1-l}}{(n-1-l) !} I_{n-1 k}\left(u\left(t_{k}\right), u^{\prime}\left(t_{k}\right), \ldots, u^{(n-1)}\left(t_{k}\right)\right), \\
& l=0,1, \ldots, n-1, \forall t \in J .
\end{aligned}
$$

So, we have

$$
\begin{aligned}
u^{(n-2)}(t)= & \int_{0}^{1} G_{n-2}(t, s) f\left(u(s), u^{\prime}(s), \ldots, u^{(n-1)}(s),(T u)(s),(S u)(s)\right) \mathrm{d} s \\
& +\sum_{0<t_{k}<t} I_{n-2 k}\left(u\left(t_{k}\right), u^{\prime}\left(t_{k}\right), \ldots, u^{(n-1)}\left(t_{k}\right)\right) \\
& +t \sum_{k=1}^{m} I_{n-1 k}\left(u\left(t_{k}\right), u^{\prime}\left(t_{k}\right), \ldots, u^{(n-1)}\left(t_{k}\right)\right) \\
& -\sum_{0<t_{k}<t}\left(t-t_{k}\right) I_{n-1 k}\left(u\left(t_{k}\right), u^{\prime}\left(t_{k}\right), \ldots, u^{(n-1)}\left(t_{k}\right)\right), \quad \forall t \in J
\end{aligned}
$$

and

$$
\begin{aligned}
u^{(n-1)}(t)= & \int_{t}^{1} f\left(s, u(s), u^{\prime}(s), \ldots, u^{(n-1)}(s),(T u)(s),(S u)(s)\right) \mathrm{d} s \\
& -\sum_{0<t_{k}<t} I_{n-1 k}\left(u\left(t_{k}\right), u^{\prime}\left(t_{k}\right), \ldots, u^{(n-1)}\left(t_{k}\right)\right) \\
& +\sum_{k=1}^{m} I_{n-1 k}\left(u\left(t_{k}\right), u^{\prime}\left(t_{k}\right), \ldots, u^{(n-1)}\left(t_{k}\right)\right), \quad \forall t \in J .
\end{aligned}
$$

Hence

$$
u^{(n)}(t)=-f\left(t, u(t), u^{\prime}(t), \ldots, u^{(n-1)}(t),(T u)(t),(S u)(t)\right), \quad \forall t \in J^{\prime}
$$


It follows from (48) and (50) that $u \in P C^{n-1}[J, E] \cap C^{n}\left[J^{\prime}, E\right]$. By (48), (49) and (50), we have

$$
\left.\Delta u^{(i)}\right|_{t=t_{k}}=I_{i k}\left(u\left(t_{k}\right), u^{\prime}\left(t_{k}\right), \ldots, u^{(n-1)}\left(t_{k}\right)\right) \quad(i=0,1, \ldots, n-2 ; k=1,2, \ldots, m)
$$

and

$$
\left.\Delta u^{(n-1)}\right|_{t=t_{k}}=-I_{n-1 k}\left(u\left(t_{k}\right), u^{\prime}\left(t_{k}\right), \ldots, u^{(n-1)}\left(t_{k}\right)\right) \quad(k=1,2, \ldots, m) .
$$

It is easy to see by (48) and (50)

$$
u^{(i)}(0)=\theta \quad(i=0,1, \ldots, n-2), \quad u^{(n-1)}(1)=\theta .
$$

By (51) to (54), $u$ is a solution of SBVP (1).

Theorem 3.1 Let conditions $\left(\mathrm{H}_{1}\right)$ to $\left(\mathrm{H}_{4}\right)$ be satisfied. Assume that

$$
\begin{aligned}
\beta= & \max \left\{\int_{0}^{1} 2 s\left(\sum_{i=0}^{n-1} L_{i}(s)+L_{n}(s) k^{*}+L_{n+1}(s) h^{*}\right) \mathrm{d} s+\sum_{k=1}^{m} \sum_{i=0}^{n-2} \frac{\left(1-t_{k}\right)^{i}}{i !} \sum_{j=0}^{n-1} M_{i k j}\right. \\
& \left.+\sum_{k=1}^{m} \sum_{j=0}^{n-1} M_{n-1 k j}, 2 \int_{0}^{1}\left(\sum_{i=0}^{n-1} L_{i}(s)+L_{n}(s) k^{*}+L_{n+1}(s) h^{*}\right) \mathrm{d} s+\sum_{k=1}^{m} \sum_{j=0}^{n-1} M_{n-1 k j}\right\} \\
& <1 .
\end{aligned}
$$

Then SBVP (1) has at least a solution $u \in P C^{n-1}[J, E] \cap C^{n}\left[J^{\prime}, E\right]$.

Proof We will use Lemma 2.5 to prove our conclusion. By $\left(\mathrm{H}_{1}\right)-\left(\mathrm{H}_{3}\right)$, from Lemma 3.2, we know $A(Q) \subset Q$.

We affirm that $A: Q \rightarrow Q$ is continuous. In fact, let $\forall\left\{u_{l}\right\}_{l=1}^{\infty} \subset Q, u_{0} \in Q, \| u_{l}-$ $u_{0} \|_{P C^{n-1}} \rightarrow 0($ as $l \rightarrow \infty)$. From the continuity of $f$ and $I_{i k}(i=0,1, \ldots, n-1, k=1,2, \ldots, m)$ and the definition of $A$, by virtue of the Lebesgue dominated convergence theorem, we see that

$$
\left\|\left(A u_{l}\right)^{(i)}(t)-\left(A u_{0}\right)^{(i)}(t)\right\| \rightarrow 0, \quad \text { as } l \rightarrow \infty, \forall t \in J, i=0,1, \ldots, n-1 .
$$

For $\forall t \in J$ (fixed), we have $\alpha\left(\left\{\left(A u_{l}\right)^{(i)}(t)\right\}_{l=1}^{\infty}\right)=0(i=0,1, \ldots, n-1)$. We also see that $\left\{A u_{l}\right\}_{l=1}^{\infty} \subset Q \subset P C^{n-1}[J, E]$ is bounded and $\left(A u_{l}\right)^{(n-1)}$ is equicontinuous on each $J_{k}$. By Lemma 2.1 , it is easy to get

$$
\alpha_{n-1}\left(\left\{A u_{l}\right\}_{l=1}^{\infty}\right)=\max _{i=0,1, \ldots, n-1}\left\{\sup _{t \in J} \alpha\left(\left\{\left(A u_{l}\right)^{(i)}(t)\right\}_{l=1}^{\infty}\right)\right\}=0,
$$

i.e., $\left\{A u_{l}\right\}_{l=1}^{\infty}$ is a relatively compact set in $P C^{(n-1)}[J, E]$. The reduction to absurdity is used to prove that $A$ is continuous. Suppose $\lim _{l \rightarrow \infty}\left\|A u_{l}-A u_{0}\right\|_{P C^{n-1}} \neq 0$. Then $\exists \epsilon_{0}>0, \exists\left\{l_{j}\right\} \subset\{l\}$ such that

$$
\left\|A u_{l_{j}}-A u_{0}\right\|_{P C^{n-1}} \geq \epsilon_{0} .
$$


On the other hand, since $\left\{A u_{l}\right\}_{l=1}^{\infty}$ is a relatively compact set in $P C^{(n-1)}[J, E]$, there exists a subsequence of $\left\{A u_{l_{j}}\right\}_{j=1}^{\infty}$ which converges to $y \in P C^{(n-1)}[J, E]$. Without loss of generality, we may assume $\left\{A u_{l_{j}}\right\}_{j=1}^{\infty}$ itself converges to $y$, that is,

$$
\left\|A u_{l_{j}}-y\right\|_{P C^{n-1}} \rightarrow 0 \quad(\text { as } j \rightarrow \infty) .
$$

By virtue of (56), we see that $y=A u_{0}$. Obviously, this is in contradiction to (58). Hence,

$$
\left\|A u_{l}-A u_{0}\right\|_{P C^{n-1}} \rightarrow 0 \quad(\text { as } l \rightarrow \infty)
$$

Consequently, $A: Q \rightarrow Q$ is continuous.

By Lemma 2.4, for any countable $B \subset Q$, which satisfies $\bar{B}=\overline{\mathrm{co}}\left(\left\{u_{0}\right\} \cup A(B)\right)$, one can see

$$
\overline{B^{(l)}(t)}=\overline{\operatorname{co}}\left(\left\{u_{0}^{(l)}(t)\right\} \cup(A(B))^{(l)}(t)\right) \quad(l=0,1, \ldots, n-1) .
$$

By virtue of the character of noncompactness, it is easy to get $\alpha_{n-1}(B)=\alpha_{n-1}(A(B))$,

$$
\begin{aligned}
\alpha\left(B^{(l)}(t)\right) & =\alpha\left(\overline{B^{(l)}(t)}\right)=\alpha\left(\overline{\operatorname{co}}\left(\left\{u_{0}^{(l)}(t)\right\} \cup(A(B))^{(l)}(t)\right)\right) \\
& =\alpha\left(\left(\left\{u_{0}^{(l)}(t)\right\} \cup(A(B))^{(l)}(t)\right)\right)=\alpha\left((A(B))^{(l)}(t)\right) \quad(l=0,1, \ldots, n-1) .
\end{aligned}
$$

For any fixed $t \in J, l=0,1, \ldots, n-2$, by condition $\left(\mathrm{H}_{4}\right)$ and Lemma 3.3, we have

$$
\begin{aligned}
\alpha\left((A(B))^{(l)}(t)\right) \leq & \int_{0}^{1} 2 s\left(\sum_{i=0}^{n-1} L_{i}(s) \alpha\left(B^{(i)}(s)\right)+L_{n}(s) k^{*} \alpha(B(s))+L_{n+1}(s) h^{*} \alpha(B(s))\right) \mathrm{d} s \\
& +\sum_{k=1}^{m} \sum_{i=l}^{n-2} \frac{\left(1-t_{k}\right)^{i-l}}{(i-l) !} \alpha\left(I_{i k}\left(B\left(t_{k}\right), B^{\prime}\left(t_{k}\right), \ldots, B^{(n-1)}\left(t_{k}\right)\right)\right) \\
& +\alpha\left(\frac{t^{n-1-l}}{(n-1-l) !} \sum_{k=1}^{m} I_{n-1 k}\left(B\left(t_{k}\right), B^{\prime}\left(t_{k}\right), \ldots, B^{(n-1)}\left(t_{k}\right)\right)\right. \\
& \left.-\frac{\left(t-t_{k}\right)^{i-l}}{(i-l) !} \sum_{0<t_{k}<t} I_{n-1 k}\left(B\left(t_{k}\right), B^{\prime}\left(t_{k}\right), \ldots, B^{(n-1)}\left(t_{k}\right)\right)\right) \\
\leq & \int_{0}^{1} 2 s\left(\sum_{i=0}^{n-1} L_{i}(s) \alpha\left(B^{(i)}(s)\right)+L_{n}(s) k^{*} \alpha(B(s))+L_{n+1}(s) h^{*} \alpha(B(s))\right) \mathrm{d} s \\
& +\sum_{k=1}^{m} \sum_{i=0}^{n-2} \frac{\left(1-t_{k}\right)^{i}}{i !} \sum_{j=0}^{n-1} M_{i k j} \alpha\left(B_{j}^{\prime}\left(t_{k}\right)\right)+\sum_{k=1}^{m} \sum_{j=0}^{n-1} M_{n-1 k j} \alpha\left(B_{j}^{\prime}\left(t_{k}\right)\right) . \quad(62)
\end{aligned}
$$

Similarly, by Lemma 3.3, for $l=n-1$, we have

$$
\begin{aligned}
& \alpha\left((A(B))^{(n-1)}(t)\right) \\
& \leq \int_{0}^{1}\left(\sum_{i=0}^{n-1} L_{i}(s) \alpha\left(B^{(i)}(s)\right)+L_{n}(s) k^{*} \alpha(B(s))+L_{n+1}(s) h^{*} \alpha(B(s))\right) \mathrm{d} s \\
& \quad+\sum_{k=1}^{m} \sum_{j=0}^{n-1} M_{n-1 k j} \alpha\left(B_{j}^{\prime}\left(t_{k}\right)\right) .
\end{aligned}
$$


Let $m^{*}=\max _{l=0,1, \ldots, n-1}\left\{\sup _{t \in J} \alpha\left((A(B))^{(l)}(t)\right)\right\}$. It is clear that $m^{*} \geq 0$. By (61) and (62), for $l=0,1, \ldots, n-2$, it is easy to see that

$$
\begin{aligned}
m^{*} \leq & \left(\int_{0}^{1} 2 s\left(\sum_{i=0}^{n-1} L_{R i}(s)+L_{R n}(s) k^{*}+L_{R n+1}(s) h^{*}\right) \mathrm{d} s\right. \\
& \left.+\sum_{k=1}^{m} \sum_{i=0}^{n-2} \frac{\left(1-t_{k}\right)^{i}}{i !} \sum_{j=0}^{n-1} M_{i k j}+\sum_{k=1}^{m} \sum_{j=0}^{n-1} M_{n-1 k j}\right) m^{*} \leq \beta m^{*} .
\end{aligned}
$$

Similarly, for $l=n-1$,

$$
m^{*} \leq\left(\int_{0}^{1} 2\left(\sum_{i=0}^{n-1} L_{i}(s)+L_{n}(s) k^{*}+L_{n+1}(s) h^{*}\right) \mathrm{d} s+\sum_{k=1}^{m} \sum_{j=0}^{n-1} M_{n-1 k j}\right) m^{*} \leq \beta m^{*}
$$

Since $\beta<1$, by (64) and (65), we know that $m^{*}=0$. It is easy to see that $A(B) \subset P C^{n-1}[J, E]$ is bounded and the elements of $(A(B))^{(n-1)}$ are equicontinuous on each $J_{k}(k=1,2, \ldots, m)$. It follows from (61) and Lemma 2.1 that

$$
\alpha_{n-1}(B)=\alpha_{n-1}(A(B))=\max _{l=0,1, \ldots, n-1}\left\{\sup _{t \in J} \alpha\left((A(B))^{(l)}(t)\right)\right\}=m^{*}=0
$$

Hence, $B$ is a relatively compact set. By Lemma 2.5 (the Mönch fixed point theorem), $A$ has at least a fixed point $u^{*} \in Q$, and by Lemma 3.4, $u^{*}$ is the solution of SBVP (1) which means conclusion holds.

An application of Theorem 3.1 is as follows.

Example Consider the following infinite system of scalar nonlinear second order impulsive integro-differential equations:

$$
\left\{\begin{aligned}
&-u_{n}^{\prime \prime}(t)= \frac{1}{n \sqrt{t(1-t)}}+\frac{t^{4}}{4 n\left(u_{2 n}(t)\right)^{\frac{1}{2}}}+\frac{t^{4}}{4} \ln \left(1+u_{n}(t)\right)+\frac{1-t^{\frac{1}{2}}}{(n+1)\left(u_{(n+1)}^{\prime}(t)\right)^{\frac{1}{3}}} \\
&+\frac{t^{3}}{4} \int_{0}^{t}(t-s) u_{n}(s) \mathrm{d} s+\frac{t^{5}}{6} \int_{0}^{1}(t+s) u_{n}(s) \mathrm{d} s, \quad 0<t<1, t \neq \frac{1}{2} \\
&\left.\triangle u_{n}\right|_{t=\frac{1}{2}}=\frac{1}{5} u_{n}\left(\frac{1}{2}\right) \\
&\left.\triangle u_{n}^{\prime}\right|_{t=\frac{1}{2}}=-\frac{1}{7} u_{n}\left(\frac{1}{2}\right)-\frac{1}{8} u_{n}^{\prime}\left(\frac{1}{2}\right), \\
& u_{n}(0)=u_{n}^{\prime}(1)=0 \quad(n=1,2, \ldots) .
\end{aligned}\right.
$$

Conclusion. The infinite system (67) has at least a $C^{2}\left(t \neq \frac{1}{2}\right)$ solution, $\left\{u_{n}(t)\right\}, u_{n}(t) \rightarrow 0$, $n \rightarrow \infty, t \neq \frac{1}{2}$.

Proof Let $J=[0,1], E=: C_{0}=\left\{u=\left(u_{1}, u_{2}, \ldots, u_{n}, \ldots\right): u_{n} \rightarrow 0\right\}$ with norm $\|u\|=\sup _{n}\left|u_{n}\right|$. We have the cone $P:=\left\{u=\left(u_{1}, \ldots, u_{n}, \ldots\right) \in C_{0}: u_{n} \geq 0, n=1,2,3, \ldots\right\}$. Obviously $P$ is a normal cone in $E$. Taking $u_{0}=\left(u_{01}, u_{02}, \ldots, u_{0 n}, \ldots\right)\left(u_{0 n}=\left(\frac{\ln \left(\frac{n}{2}+1\right)}{n}\right)^{2}\right)$, it is easy to see $u_{0} \in$ $P, 0<\left\|u_{0}\right\|=\left(\ln \frac{3}{2}\right)^{2}<1$ and $P_{1}=\left\{u \in P: u_{n} \geq u_{0 n}\|u\|\right\}$. The infinite system (67) can be regarded as a SBVP of the form (1) in $E$. In this situation,

$$
\begin{aligned}
& k(t, s)=t-s \in C\left[D, R_{+}\right] \quad(D=\{(t, s) \in J \times J: s \leq t\}), \\
& h(t, s)=t+s \in C\left[J \times J, R_{+}\right],
\end{aligned}
$$




$$
\begin{aligned}
& u=\left(u_{1}, u_{2}, \ldots, u_{n}, \ldots\right), \quad v=\left(v_{1}, v_{2}, \ldots, v_{n}, \ldots\right), \quad w=\left(w_{1}, w_{2}, \ldots, w_{n}, \ldots\right), \\
& x=\left(x_{1}, x_{2}, \ldots, x_{n}, \ldots\right), \quad f=\left(f_{1}, f_{2}, \ldots, f_{n}, \ldots\right),
\end{aligned}
$$

in which

$$
\begin{aligned}
f_{n}(t, u, v, w, x)= & \frac{1}{n \sqrt{t(1-t)}}+\frac{t^{4}}{4 n\left(u_{2 n}\right)^{\frac{1}{2}}} \\
& +\frac{t^{4}}{4} \ln \left(1+u_{n}\right)+\frac{1-t^{\frac{1}{2}}}{(n+1)\left(v_{(n+1)}\right)^{\frac{1}{3}}}+\frac{t^{3}}{4} w_{n}+\frac{t^{5}}{6} x_{n}
\end{aligned}
$$

and $t_{k}=\frac{1}{2}, I_{i k}=\left(I_{i k 1}, I_{i k 2}, \ldots, I_{i k n}, \ldots\right)(i=0,1)$, where

$$
I_{01 n}(u, v)=\frac{1}{5} u_{n}, \quad I_{11 n}(u, v)=\frac{1}{7} u_{n}+\frac{1}{8} v_{n} .
$$

Obviously, for $(t, u, v, w, x) \in(0,1) \times P_{1} \backslash\{\theta\} \times P_{1} \backslash\{\theta\} \times P_{1} \times P_{1}$, we have

$$
\begin{aligned}
& 4 n\left(u_{2 n}\right)^{\frac{1}{2}} \geq 2 \ln (n+1)(\|u\|)^{\frac{1}{2}}>0, \\
& (n+1)\left(v_{(n+1)}\right)^{\frac{1}{3}} \geq \sqrt[3]{n+1}\left(\ln \left(\frac{n+1}{2}+1\right)\right)^{\frac{2}{3}}(\|v\|)^{\frac{1}{3}}>0, \\
& w_{n} \geq\left(\frac{\ln \left(\frac{n}{2}+1\right)}{n}\right)^{2}\|w\|, \quad x_{n} \geq\left(\frac{\ln \left(\frac{n}{2}+1\right)}{n}\right)^{2}\|x\| \quad(n=1,2,3, \ldots),
\end{aligned}
$$

which implies

$$
\begin{aligned}
\left|f_{n}\right| \leq & \frac{1}{n \sqrt{t(1-t)}}+\frac{t^{4}}{2 \ln (n+1)(\|u\|)^{\frac{1}{2}}}+\frac{t^{4}}{4} \ln \left(1+u_{n}\right) \\
& +\frac{1-t^{\frac{1}{2}}}{\sqrt[3]{n+1}\left(\ln \left(\frac{n+1}{2}+1\right)\right)^{\frac{2}{3}}(\|v\|)^{\frac{1}{3}}}+\frac{t^{3}}{4} w_{n}+\frac{t^{5}}{6} x_{n} \quad(n=1,2,3, \ldots) .
\end{aligned}
$$

Since

$$
u_{n} \rightarrow 0, \quad v_{n} \rightarrow 0, \quad w_{n} \rightarrow 0, \quad x_{n} \rightarrow 0, \quad \ln (n+1) \rightarrow+\infty
$$

and

$$
\sqrt[3]{n+1}\left(\ln \left(\frac{n+1}{2}+1\right)\right)^{\frac{2}{3}} \rightarrow+\infty
$$

as $n \rightarrow+\infty$, we have

$$
\left|f_{n}\right| \rightarrow 0, \quad n \rightarrow+\infty
$$

That is, $f \in E$. Obviously, $f \in P$. By (71), we can see

$$
\begin{aligned}
\|f\| \leq & \frac{1}{\sqrt{t(1-t)}}+\frac{t^{4}}{2 \ln 2(\|u\|)^{\frac{1}{2}}}+\frac{t^{4}}{4} \ln (1+\|u\|) \\
& +\frac{1-t^{\frac{1}{2}}}{\sqrt[3]{2}(\ln 2)^{\frac{2}{3}}(\|v\|)^{\frac{1}{3}}}+\frac{t^{3}}{4}\|w\|+\frac{t^{5}}{6}\|x\| .
\end{aligned}
$$


On the other hand, from (68) and (70), we have

$$
\begin{aligned}
f_{n}(t, u, v, w, x) \geq & \frac{1}{n \sqrt{t(1-t)}}+\frac{t^{4}}{4 n(\|u\|)^{\frac{1}{2}}}+\frac{t^{4}}{4} \ln \left(1+\left(\frac{\ln \left(\frac{n}{2}+1\right)}{n}\right)^{2}\|u\|\right) \\
& +\frac{1-t^{\frac{1}{2}}}{(n+1)(\|v\|)^{\frac{1}{3}}}+\frac{t^{3}}{4}\left(\frac{\ln \left(\frac{n}{2}+1\right)}{n}\right)^{2}\|w\|+\frac{t^{5}}{6}\left(\frac{\ln \left(\frac{n}{2}+1\right)}{n}\right)^{2}\|x\| \\
\geq & \left(\frac{\ln \left(\frac{n}{2}+1\right)}{n}\right)^{2}\left(\frac{n}{\ln ^{2}\left(\frac{n}{2}+1\right) \sqrt{t(1-t)}}\right. \\
& +\frac{n t^{4}}{4 \ln ^{2}\left(\frac{n}{2}+1\right)(\|u\|)^{\frac{1}{2}}}+\frac{t^{4}}{4} \ln (1+\|u\|) \\
& \left.+\frac{n^{2}\left(1-t^{\frac{1}{2}}\right)}{(n+1) \ln ^{2}\left(\frac{n}{2}+1\right)(\|v\|)^{\frac{1}{3}}}+\frac{t^{3}}{4}\|w\|+\frac{t^{5}}{6}\|x\|\right) \quad(n=1,2,3, \ldots) .
\end{aligned}
$$

It is easy to get

$$
n \ln 2 \geq 2 \ln ^{2}\left(\frac{n}{2}+1\right), \quad n^{2} \sqrt[3]{2} \ln ^{\frac{2}{3}} 2 \geq(n+1) \ln ^{2}\left(\frac{n}{2}+1\right) \quad(n=1,2,3, \ldots) .
$$

It follows from (72), (73) and (74) that

$$
\begin{aligned}
f_{n}(t, u, v, w, x) \geq & \left(\frac{\ln \left(\frac{n}{2}+1\right)}{n}\right)^{2}\left(\frac{1}{\sqrt{t(1-t)}}+\frac{t^{4}}{2 \ln 2(\|u\|)^{\frac{1}{2}}}\right. \\
& \left.+\frac{t^{4}}{4} \ln (1+\|u\|)+\frac{1-t^{\frac{1}{2}}}{\sqrt[3]{2}(\ln 2)^{\frac{2}{3}}(\|v\|)^{\frac{1}{3}}}+\frac{t^{3}}{4}\|w\|+\frac{t^{5}}{6}\|x\|\right) \\
\geq & \left(\frac{\ln \left(\frac{n}{2}+1\right)}{n}\right)^{2}\|f\|=u_{0 n}\|f\| \quad(n=1,2,3, \ldots) .
\end{aligned}
$$

Hence, $f \in C\left[(0,1) \times P_{1} \backslash\{\theta\} \times P_{1} \backslash\{\theta\} \times P_{1} \times P_{1}, P_{1}\right]$. Similarly, we have $I_{01}, I_{11} \in C\left[P_{1} \times\right.$ $\left.P_{1}, P_{1}\right]$.

Taking

$$
\begin{aligned}
& b(t)=\frac{1}{\sqrt{t(1-t)}}, \quad a_{0}(t)=\frac{t^{4}}{2}, \quad a_{1}(t)=1-t^{\frac{1}{2}}, \quad a_{2}(t)=\frac{t^{3}}{4}, \quad a_{3}(t)=\frac{t^{5}}{6}, \\
& g_{0}(y)=\frac{1}{\ln 2 y^{\frac{1}{2}}}, \quad g_{1}(y)=\frac{1}{\sqrt[3]{2}(\ln 2)^{\frac{2}{3}} y^{\frac{1}{3}}}, \quad g_{2}(y)=g_{3}(y)=y
\end{aligned}
$$

and

$$
h_{0}(y)=\frac{1}{2} \ln (1+y), \quad h_{1}(y)=0,
$$

by (69) and (72), condition $\left(\mathrm{H}_{1}\right)$ holds.

For any $u \in P_{1}$, define $\varphi$ by $\varphi(u)=u_{1}$. It is easy to see $\varphi \in P_{1}^{*},\|\varphi\|=1$ and $\varphi\left(u_{0}\right)=\ln ^{2} \frac{3}{2}$. For any $r>0$, let

$$
h_{r}(t)=\frac{1}{\sqrt{t(1-t)}}
$$


Therefore, condition $\left(\mathrm{H}_{2}\right)$ is satisfied. It follows from (68) that

$$
\begin{aligned}
& \varphi(f(t, u, v, w, x))=f_{1}(t, u, v, w, x) \geq \frac{1}{\sqrt{t(1-t)}}=h_{r}(t), \\
& t \in(0,1), u, v \in P_{1 r} \backslash\{\theta\}, w, x \in P_{1 r} .
\end{aligned}
$$

Now, we check that condition $\left(\mathrm{H}_{3}\right)$ is true. In fact, it is easy to get

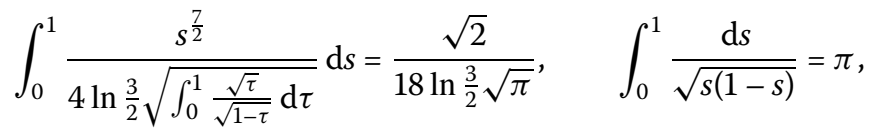

$$
\begin{aligned}
& \int_{0}^{1} \frac{1-s^{\frac{1}{2}}}{\sqrt[3]{2}\left(\ln 2 \ln \frac{3}{2}\right)^{\frac{2}{3}}\left(\int_{s}^{1} \frac{1}{\sqrt{\tau(1-\tau)}} \mathrm{d} \tau\right)^{\frac{1}{3}}} \mathrm{~d} s \leq\left(\frac{2}{\ln 2 \ln \frac{3}{2}}\right)^{\frac{2}{3}}
\end{aligned}
$$

with $k^{*}=1$ and $h^{*}=2$. Since

$$
\frac{\sqrt{2}}{18 \ln \frac{3}{2} \sqrt{\pi}}+\frac{1}{16}+\frac{1}{15}+\frac{1}{5}+\frac{1}{7}+\frac{1}{8}<1
$$

there exists a sufficient large $R_{0}>0$ such that

$$
\begin{aligned}
& \int_{0}^{1}\left(\frac{1}{\sqrt{s(1-s)}}+\frac{s^{4}}{2 \ln 2 \ln \frac{3}{2} \sqrt{\int_{0}^{1} \frac{\sqrt{\tau}}{\sqrt{1-\tau}} \mathrm{d} \tau}}\left(1+\frac{\ln 2}{2} R_{0}^{\frac{1}{2}} \ln \left(1+R_{0}\right)\right)\right. \\
& \left.+\frac{1-s^{\frac{1}{2}}}{\sqrt[3]{2}\left(\ln 2 \ln \frac{3}{2}\right)^{\frac{2}{3}}\left(\int_{s}^{1} \frac{1}{\sqrt{\tau(1-\tau)}} \mathrm{d} \tau\right)^{\frac{1}{3}}}+\frac{s^{3}}{4} R_{0}+\frac{s^{5}}{6} 2 R_{0}\right) \mathrm{d} s+\left(\frac{1}{5}+\frac{1}{7}+\frac{1}{8}\right) R_{0}<R_{0}
\end{aligned}
$$

which implies that condition $\left(\mathrm{H}_{3}\right)$ is satisfied.

Let

$$
f=f^{1}+f^{2}+f^{3}+f^{4}
$$

in which

$$
\begin{aligned}
& f^{1}=\left(f_{1}^{1}, f_{2}^{1}, \ldots, f_{n}^{1}, \ldots\right), \quad f^{2}=\left(f_{1}^{2}, f_{2}^{2}, \ldots, f_{n}^{2}, \ldots\right), \\
& f^{3}=\left(f_{1}^{3}, f_{2}^{3}, \ldots, f_{n}^{3}, \ldots\right), \quad f^{4}=\left(f_{1}^{4}, f_{2}^{4}, \ldots, f_{n}^{4}, \ldots\right),
\end{aligned}
$$

where

$$
\begin{aligned}
& f_{n}^{1}(t, u, v, w, x)=\frac{1}{n \sqrt{t(1-t)}}+\frac{t^{4}}{4 n\left(u_{2 n}\right)^{\frac{1}{2}}}+\frac{1-t^{\frac{1}{2}}}{(n+1)\left(v_{(n+1)}\right)^{\frac{1}{3}}} \\
& f_{n}^{2}(t, u, v, w, x)=\frac{t^{4}}{4} \ln \left(1+u_{n}\right), \\
& f_{n}^{3}(t, u, v, w, x)=\frac{t^{3}}{4} w_{n}, f_{n}^{4}(t, u, v, w, x)=\frac{t^{5}}{6} x_{n} .
\end{aligned}
$$


For any

$$
\begin{gathered}
b>a>0, \quad z=\left(z_{1}, z_{2}, \ldots, z_{n}, \ldots\right) \in f^{1}\left(t, B_{0}, B_{1}, B_{2}, B_{3}\right) \\
\left(\forall t \in(0,1), B_{0}, B_{1} \subset \overline{P_{1 b}} \backslash P_{1 a}, B_{2}, B_{3} \subset \overline{P_{1 b}}\right),
\end{gathered}
$$

by (71) and (78), it is easy to get

$$
\left|z_{n}\right| \leq \frac{1}{n \sqrt{t(1-t)}}+\frac{t^{4}}{4 n a^{\frac{1}{2}}}+\frac{1-t^{\frac{1}{2}}}{(n+1) a^{\frac{1}{3}}}, \quad n=1,2, \ldots
$$

Hence, the relative compactness of $f^{1}\left(t, B_{0}, B_{1}, B_{2}, B_{3}\right)$ in $C_{0}$ follows directly from a known result (see [14]): a bounded set $X$ of $C_{0}$ is relatively compact if and only if

$$
\lim _{n \rightarrow \infty}\left\{\sup _{z \in X}\left[\max \left\{\left|Z_{k}\right|: k \geq n\right\}\right]\right\}=0 .
$$

That is,

$$
\alpha\left(f^{1}\left(t, B_{0}, B_{1}, B_{2}, B_{3}\right)\right)=0, \quad \forall t \in(0,1), B_{0}, B_{1} \subset \overline{P_{1 b}} \backslash P_{1 a}, B_{2}, B_{3} \subset \overline{P_{1 b}} .
$$

For any

$$
b>a>0, \quad(t, u, v, w, x),(t, \bar{u}, \bar{v}, \bar{w}, \bar{x}) \in(0,1) \times \overline{P_{1 b}} \backslash P_{1 a} \times \overline{P_{1 b}} \backslash P_{1 a} \times \overline{P_{1 b}} \times \overline{P_{1 b}},
$$

by (78), one can get

$$
\left|f_{n}^{2}(t, u, v, w, x)-f_{n}^{2}(t, \bar{u}, \bar{v}, \bar{w}, \bar{x})\right|=\frac{t^{4}}{4}\left|\ln \left(1+u_{n}\right)-\ln \left(1+\overline{u_{n}}\right)\right| \leq \frac{t^{4}}{4} \frac{\left|u_{n}-\overline{u_{n}}\right|}{1+\xi_{n}},
$$

in which $\xi_{n} \in\left(u_{n}, \overline{u_{n}}\right)$. Since $u_{n} \geq u_{0 n} a>0$ and $\overline{u_{n}} \geq u_{0 n} a>0$, by (81), it is easy to see

$$
\begin{aligned}
& \left\|f^{2}(t, u, v, w, x)-f^{2}(t, \bar{u}, \bar{v}, \bar{w}, \bar{x})\right\| \leq \frac{t^{4}}{4}\|u-\bar{u}\|, \\
& \quad(t, u, v, w, x),(t, \bar{u}, \bar{v}, \bar{w}, \bar{x}) \in(0,1) \times \overline{P_{1 b}} \backslash P_{1 a} \times \overline{P_{1 b}} \backslash P_{1 a} \times \overline{P_{1 b}} \times \overline{P_{1 b}},
\end{aligned}
$$

which implies

$$
\alpha\left(f^{2}\left(t, B_{0}, B_{1}, B_{2}, B_{3}\right)\right) \leq \frac{t^{4}}{4} \alpha\left(B_{0}\right), \quad \forall t \in(0,1), B_{0}, B_{1} \subset \overline{P_{1 b}} \backslash P_{1 a}, B_{2}, B_{3} \subset \overline{P_{1 b}} .
$$

Similarly, by (78),

$$
\alpha\left(f^{3}\left(t, B_{0}, B_{1}, B_{2}, B_{3}\right)\right) \leq \frac{t^{3}}{4} \alpha\left(B_{2}\right), \quad \forall t \in(0,1), B_{0}, B_{1} \subset \overline{P_{1 b}} \backslash P_{1 a}, B_{2}, B_{3} \subset \overline{P_{1 b}}
$$

and

$$
\alpha\left(f^{4}\left(t, B_{0}, B_{1}, B_{2}, B_{3}\right)\right) \leq \frac{t^{5}}{6} \alpha\left(B_{3}\right), \quad \forall t \in(0,1), B_{0}, B_{1} \subset \overline{P_{1 b}} \backslash P_{1 a}, B_{2}, B_{3} \subset \overline{P_{1 b}} .
$$


By (80), (82), (83) and (84), it is easy to get

$$
\begin{aligned}
\alpha\left(f\left(t, B_{0}, B_{1}, B_{2}, B_{3}\right)\right) \leq & \alpha\left(f^{1}\left(t, B_{0}, B_{1}, B_{2}, B_{3}\right)\right)+\alpha\left(f^{2}\left(t, B_{0}, B_{1}, B_{2}, B_{3}\right)\right) \\
& +\alpha\left(f^{3}\left(t, B_{0}, B_{1}, B_{2}, B_{3}\right)\right)+\alpha\left(f^{4}\left(t, B_{0}, B_{1}, B_{2}, B_{3}\right)\right) \\
\leq & \frac{t^{4}}{4} \alpha\left(B_{0}\right)+\frac{t^{3}}{4} \alpha\left(B_{2}\right)+\frac{t^{5}}{6} \alpha\left(B_{3}\right), \\
& \forall t \in(0,1), B_{0}, B_{1} \subset \overline{P_{1 b}} \backslash P_{1 a}, B_{2}, B_{3} \subset \overline{P_{1 b}} .
\end{aligned}
$$

In the same way,

$$
\begin{aligned}
& \alpha\left(I_{01}\left(B_{0}^{\prime}, B_{1}^{\prime}\right)\right) \leq \frac{1}{5} \alpha\left(B_{0}^{\prime}\right), \\
& \alpha\left(I_{11}\left(B_{0}^{\prime}, B_{1}^{\prime}\right)\right) \leq \frac{1}{7} \alpha\left(B_{0}^{\prime}\right)+\frac{1}{8} \alpha\left(B_{1}^{\prime}\right), \quad \forall B_{0}^{\prime}, B_{1}^{\prime} \subset \overline{P_{1 b}} .
\end{aligned}
$$

Taking

$$
\begin{aligned}
& L_{0}(t)=\frac{t^{4}}{4}, \quad L_{1}(t) \equiv 0, \quad L_{2}(t)=\frac{t^{3}}{4}, \quad L_{3}(t)=\frac{t^{5}}{6}, \\
& M_{010}=\frac{1}{5}, \quad M_{011}=0, \quad M_{110}=\frac{1}{7}, \quad M_{111}=\frac{1}{8},
\end{aligned}
$$

the condition $\left(\mathrm{H}_{4}\right)$ follows from (86) and (87). We can calculate and get

$$
\beta=\max \left\{\frac{1}{12}+\frac{1}{10}+\frac{2}{21}+\frac{1}{5}+\frac{1}{7}+\frac{1}{8}, \frac{1}{10}+\frac{1}{8}+\frac{1}{9}+\frac{1}{7}+\frac{1}{8}\right\}<1 .
$$

Therefore, by Theorem 3.1, the conclusion holds.

\section{Competing interests}

The authors declare that they have no competing interests.

\section{Authors' contributions}

All authors typed, read and approved the final manuscript.

\section{Author details}

${ }^{1}$ School of Economics, Shandong University, Jinan, Shandong 250100, People's Republic of China. ${ }^{2}$ Department of Mathematics, Qilu Normal University, Jinan, Shandong 250013, People's Republic of China.

\section{Acknowledgements}

The authors would like to thank the reviewers for carefully reading this article and making valuable comments and suggestions. The project is supported by the National Natural Science Foundation of P.R. China (71272119), Social Science Foundation of Shandong Province (13CJRJ07) and Teaching and Research Projects of Qi Lu Normal University (201306).

Received: 2 January 2014 Accepted: 6 May 2014 Published: 20 May 2014

\section{References}

1. Guo, D: Existence of solutions for $n$ th-order impulsive integro-differential equations in a Banach space. Nonlinear Anal. 47, 741-752 (2001)

2. Chen, Y, Qin, B: The multiple solutions for a class of singular boundary value problems of nonlinear integro-differential equations in Banach spaces. J. Syst. Sci. Math. Sci. 25(5), 550-561 (2005)

3. Liu, L, Hao, X, Wu, Y: Positive solutions for singular second order differential equations with integral boundary conditions. Math. Comput. Model. 57(3-4), 836-847 (2013)

4. Zhang, $X$ : Existence of positive solution for second-order nonlinear impulsive singular differential equations of mixed type in Banach spaces. Nonlinear Anal. 70, 1620-1628 (2009) 
5. Chen, Y, Qin, B: Multiple positive solutions for first-order impulsive singular integro-differential equations on the half line in a Banach space. Bound. Value Probl. 2013, 69 (2013). doi:10.1186/1687-2770-2013-69

6. Chen, Y: Multiple solutions for boundary value problems of $n$ th-order nonlinear integrodifferential equations in Banach spaces. Abstr. Appl. Anal. 2013, Article ID 296038 (2013). doi:10.1155/2013/296038

7. Guo, D: Existence of positive solutions for $n$ th-order nonlinear impulsive singular integro-differential equations in Banach spaces. Nonlinear Anal. 68, 2727-2740 (2008)

8. Guo, D: Positive solutions of an infinite boundary value problem for $n$ th-order nonlinear impulsive singular integro-differential equations in Banach spaces. Nonlinear Anal. 70, 2078-2090 (2009)

9. Liu, Y: Positive solutions of second order singular initial value problem in Banach spaces. Indian J. Pure Appl. Math. 33(6), 833-845 (2002)

10. Guo, D, Lakshmikantham, V, Liu, XZ: Nonlinear Integral Equation in Abstract Spaces. Kluwer Academic, Dordrecht (1996)

11. Hein, HP: On the behaviour of measure of noncompactness with respect to differentiation and integration of vector-valued functions. Nonlinear Anal. 7, 1351-1371 (1983)

12. Zhang, $X$, Sun, J: Solutions of nonlinear second-order impulsive integrodifferential equations of mixed type in Banach spaces. J. Syst. Sci. Math. Sci. 22(4), 428-438 (2002)

13. Deimling, K: Nonlinear Functional Analysis. Springer, Berlin (1985)

14. Banas, J, Goebel, K: Measure of Noncompactness in Banach Spaces. Dekker, New York (1980)

10.1186/1687-2770-2014-128

Cite this article as: Chen et al.: Solutions for $n$ th-order boundary value problems of impulsive singular nonlinear integro-differential equations in Banach spaces. Boundary Value Problems 2014, 2014:128

\section{Submit your manuscript to a SpringerOpen ${ }^{0}$ journal and benefit from:}

- Convenient online submission

- Rigorous peer review

- Immediate publication on acceptance

Open access: articles freely available online

- High visibility within the field

- Retaining the copyright to your article 